\title{
RELICS: The Reionization Lensing Cluster Survey and the Brightest High- $z$ Galaxies
}

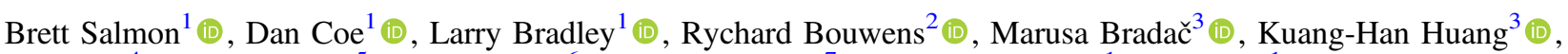
Pascal A. Oesch ${ }^{4}$ (D), Daniel Stark ${ }^{5}$, Keren Sharon ${ }^{6}$ (D) , Michele Trenti ${ }^{7}$ (10), Roberto J. Avila ${ }^{1}$, Sara Ogaz ${ }^{1}$, Felipe Andrade-Santos ${ }^{8}$ (D), Daniela Carrasco ${ }^{7}$, Catherine Cerny ${ }^{6}$, William Dawson ${ }^{9}$ (i), Brenda L. Frye ${ }^{5}$, Austin Hoag ${ }^{10}$ (1) , Traci Lin Johnson ${ }^{6}$ (1), Christine Jones ${ }^{8}$, Daniel Lam $^{2}$ (D), Lorenzo Lovisari ${ }^{8}$, Ramesh Mainali ${ }^{5}$ (10), Matt Past ${ }^{6}$, Rachel Paterno-Mahler ${ }^{6}$ (10), Avery Peterson ${ }^{6}$, Adam G. Riess ${ }^{1,11}$, Steven A. Rodney ${ }^{12}$ (1) , Russel E. Ryan ${ }^{1}$, Irene Sendra-Server ${ }^{13}$, Victoria Strait ${ }^{3}$ (10), Louis-Gregory Strolger ${ }^{1}$, Keiichi Umetsu ${ }^{14}$ (1), Benedetta Vulcani ${ }^{15}$ (i), and Adi Zitrin ${ }^{16}$ (1)

${ }^{1}$ Space Telescope Science Institute, Baltimore, MD, USA; bsalmon@stsci.edu

${ }^{2}$ Leiden Observatory, Leiden University, NL-2300 RA Leiden, The Netherlands

${ }^{3}$ Department of Physics, University of California, Davis, CA 95616, USA

${ }^{4}$ Geneva Observatory, University of Geneva, Ch. des Maillettes 51,1290 Versoix, Switzerland

${ }^{5}$ Department of Astronomy, Steward Observatory, University of Arizona, 933 North Cherry Avenue, Room N204, Tucson, AZ 85721, USA

${ }^{6}$ Department of Astronomy, University of Michigan, 1085 South University Avenue, Ann Arbor, MI 48109, USA

School of Physics, University of Melbourne, VIC 3010, Australia

${ }_{9}^{8}$ Harvard-Smithsonian Center for Astrophysics, 60 Garden Street, Cambridge, MA 02138, USA

${ }^{9}$ Lawrence Livermore National Laboratory, P.O. Box 808 L- 210, Livermore, CA 94551, USA

${ }^{10}$ Department of Physics and Astronomy, University of California, Los Angeles, CA 90095-1547, USA

${ }^{11}$ Department of Physics and Astronomy, The Johns Hopkins University, Baltimore, MD 21218, USA

${ }_{12}^{12}$ Department of Physics and Astronomy, University of South Carolina, 712 Main Street, Columbia, SC 29208, USA

${ }^{13}$ Infrared Processing and Analysis Center, California Institute of Technology, MS 100-22, Pasadena, CA 91125, USA

${ }^{14}$ Institute of Astronomy and Astrophysics, Academia Sinica, PO Box 23-141, Taipei 10617, Taiwan

${ }^{15}$ INAF- Osservatorio astronomico di Padova, Vicolo Osservatorio 5, I-35122 Padova, Italy

${ }^{16}$ Physics Department, Ben-Gurion University of the Negev, P.O. Box 653, Beer-Sheva 84105, Israel

Received 2019 May 31; revised 2019 July 30; accepted 2019 July 31; published 2020 February 5

\begin{abstract}
Massive foreground galaxy clusters magnify and distort the light of objects behind them, permitting a view into both the extremely distant and intrinsically faint galaxy populations. We present here the $z \sim 6-8$ candidate highredshift galaxies from the Reionization Lensing Cluster Survey (RELICS), a Hubble and Spitzer Space Telescope survey of 41 massive galaxy clusters spanning an area of $\approx 200 \mathrm{arcmin}^{2}$. These clusters were selected to be excellent lenses, and we find similar high-redshift sample sizes and magnitude distributions as the Cluster Lensing And Supernova survey with Hubble (CLASH). We discover 257, 57, and eight candidate galaxies at $z \sim 6$, 7, and 8 respectively, (322 in total). The observed (lensed) magnitudes of the $z \sim 6$ candidates are as bright as AB mag $\sim 23$, making them among the brightest known at these redshifts, comparable with discoveries from much wider, blank-field surveys. RELICS demonstrates the efficiency of using strong gravitational lenses to produce highredshift samples in the epoch of reionization. These brightly observed galaxies are excellent targets for follow-up study with current and future observatories, including the James Webb Space Telescope.
\end{abstract}

Key words: Galaxies - galaxies: high-redshift

Supporting material: machine-readable tables

\section{Introduction}

Images from modern extragalactic surveys are rich with red sources as we push deeper to reveal the faint, redshifted population of the very first galaxies. Our investment in this early epoch is for good reason; the first billion years of the universe $\left(t_{\text {universe }} \approx 1 \mathrm{Gyr}\right.$ at $z=5.5$ ) cover an era of rapid evolution by both the first stars and the first galaxies (for a complete review, see Stark 2016). Moreover, this period spans the time when the universe underwent a phase transition from having primarily neutral hydrogen to primarily ionized hydrogen, a process called reionization. Understanding the properties and the relative number of intrinsically faint and intrinsically bright galaxies at this epoch directly affects our interpretation of how reionization occurred, given that the most likely culprits for reionization were intrinsically faint galaxies at $z>6$ (Madau et al. 1999; Yan et al. 2003; Bunker et al. 2004; Oesch et al. 2009; Finkelstein et al. 2012; Kuhlen \& FaucherGiguère 2012; McLure et al. 2013; Schmidt et al. 2014; Atek et al. 2015a; Ishigaki et al. 2015; Robertson et al. 2015; Matthee et al. 2017; Lam et al. 2019).
There have been a variety of approaches to reach this distant galaxy population. While more costly, deep and wide spacebased, "blank-field" surveys such as the Cosmic Assembly Deep Extragalactic Legacy Survey (CANDELS; Grogin et al. 2011; Koekemoer et al. 2011) and the Hubble Ultra Deep Field (HUDF; Beckwith et al. 2006; Bouwens et al. 2011; Ellis et al. 2013; Illingworth et al. 2013; Koekemoer et al. 2013) and Brightest of Reionization Galaxies (BoRG; Trenti et al. 2011; Bradley et al. 2012; Livermore et al. 2018) Survey have produced exquisite data sets that have become a reference data set for high-redshift galaxies. The same approach with ground-based surveys, such as UltraVista (Scoville et al. 2007; McCracken et al. 2012; Bowler et al. 2012, 2017), have similarly produced copious numbers of intrinsically bright high- $z$ galaxies. Similarly, thousands of bright galaxies at $z=6-7$ were found in the Great Optically Luminous Dropout Research Using Subaru HSC (GOLDRUSH; Harikane et al. 2018; Ono et al. 2018) and the Systematic Identification of LAEs for Visible Exploration and Reionization Research Using Subaru HSC (SILVERRUSH; Konno et al. 2018; Ouchi et al. 2018; Shibuya et al. 2018a, 2018b) surveys, confirming the low- $z$ 
relation that galaxies with high equivalent width from restultraviolet (UV) nebular lines, including Ly $\alpha 1216 \AA$, are also metal poor and faint in their rest-UV continuum. This approach of searching deep or wide on "blank fields" has proven lucrative and has lead to the discovery of the inexplicably bright, most distant confirmed galaxy found to date at $z=11.1$ ( $t_{\text {universe }} \approx 400 \mathrm{Myr}$; Oesch et al. 2016).

Another approach is to take advantage of natural telescopes by observing strong gravitational lenses. Cluster-lensing surveys such as the Cluster Lensing and Supernovae Survey with Hubble (CLASH; Postman et al. 2012; Zheng et al. 2012; Coe et al. 2013; Bouwens et al. 2014; Bradley et al. 2014) and the Hubble Frontier Fields (HFF; Atek et al. 2015b; Coe et al. 2015; Lotz et al. 2017; Ishigaki et al. 2018) have produced most of the $z \geqslant 8$ galaxy candidates and allowed us to make the first inferences of the star formation rate density at $z=9-10$ (Zitrin et al. 2014; Oesch et al. 2015). Moreover, the magnifications produced by lensing enables us to reach intrinsically faint, low-mass galaxies. Thanks to carefully calibrated lensing models (e.g., Meneghetti et al. 2017), subtraction of intracluster light (Merlin et al. 2016; Livermore et al. 2017), and calibration of the measured sizes (Kawamata et al. 2015; Bouwens et al. 2017a), there has been substantial progress in deriving both the prevalence of intrinsically faint, lower-luminosity galaxies and the faint-end slope of the ultraviolet (UV) luminosity function (LF; Atek et al. 2014; Alavi et al. 2016; Bouwens et al. 2017b).

In addition, it is important to find highly magnified galaxies to detect intrinsically faint UV metal lines, such as C IV $\lambda 1548 \AA$ (Stark et al. 2014, 2015b) and C III] $\lambda 1909 \AA$ (Rigby et al. 2015; Stark et al. 2015a, 2017; Mainali et al. 2017) at high redshift. These UV lines can now be seen out to $z=6-7$, including intrinsically faint $\operatorname{Ly} \alpha$ (Hoag et al. 2017; Hashimoto et al. 2018). It is imperative to detect these faint metal lines not only because they help us to deduce the shape of the ionizing spectra, but they also allow us to spectroscopically confirm the redshifts of galaxies in the epoch of reionization, given that the Ly $\alpha$ line becomes completely opaque to the line-of-site neutral intergalactic medium (Stark et al. 2010; Schenker et al. 2012; Tilvi et al. 2013; Pentericci et al. 2014).

The rich history of using strong lensing systems to study in detail $z \approx 4-7$ galaxies (Franx et al. 1997; Bradley et al. 2008; Zitrin et al. 2012; Jones et al. 2013) and reveal the $z \approx 8-11$ population (Zheng et al. 2012; Bouwens et al. 2014; Zitrin et al. 2014; McLeod et al. 2016; Ishigaki et al. 2018) was the motivation for the Reionization Lensing Cluster Survey (RELICS; Coe et al. 2019). RELICS is a 190-orbit Hubble Space Telescope (HST) Treasury Program designed to build off of the success of other HST lensing surveys like CLASH and the HFF, and take advantage of clusters with existing HST/ ACS imaging and/or data suggesting exceptionally high cluster masses. In short, the survey targeted 41 massive galaxy clusters selected by the Planck survey (Planck Collaboration et al. 2016) to be excellent lensing systems. This survey is timely in advance of the James Webb Space Telescope (JWST) launch date, as JWST was not designed to be a wide-field survey telescope and will benefit from existing high-redshift candidates. We present here the first results of the RELICS program [10.17909/T9SP45 ], providing to the community all of its high-redshift candidates found to date.
This paper is organized as follows. In Section 2, we summarize our observations, redshifts, and selection. In Section 3, we describe our resulting magnitudes of the objects in our sample, and present the spectral energy distributions (SEDs) and images of bright sources. In Section 4, we discuss our conclusions and future work. Throughout, we assume concordance cosmology using $H_{0}=$ $70 \mathrm{~km} \mathrm{~s}^{-1} \mathrm{Mpc}^{-1}, \Omega_{\mathrm{M}, 0}=0.3$ and $\Omega_{\Lambda, 0}=0.7$. All magnitudes quoted here are measured with respect to the $\mathrm{AB}$ system, $m_{\mathrm{AB}}=$ 31.4-2.5 $\log \left(f_{\nu} / 1\right.$ nJy) (Oke \& Gunn 1983).

\section{Data, Redshifts, and Sample Selection}

\subsection{RELICS Cluster Selection and HST Photometry}

The full details of the selection criteria for the RELICS cluster fields are described by Coe et al. (2019). In short, the clusters were selected by a combination of their cluster mass and preexisting ACS imaging. From the most massive Planck clusters (identified by their Sunyaev Zel'dovich cluster mass; Planck Collaboration et al. 2016), we first selected the eight most massive clusters that had HST/ACS but not WFC3 infrared imaging, and another 13 massive Planck clusters that had no HST or Spitzer imaging at all. The 20 other RELICS clusters are selected from known strong lenses that already have HST optical imaging. We also note that seven of the RELICS clusters can be found in the MACS program by Ebeling et al. (2001). We initially inferred the cluster-lensing strengths from a variety of sources, including their X-ray mass (MCXC; Mantz et al. 2010; Piffaretti et al. 2011), weak lensing mass (Applegate et al. 2014; Umetsu et al. 2014; von der Linden et al. 2014; Hoekstra et al. 2015; Sereno 2015), Sloan Digital Sky Survey data (Wen et al. 2012; Wong et al. 2013), and other SZ mass estimates (Hasselfield et al. 2013; Bleem et al. 2015). Further details on the cluster selection can be found in Cerny et al. (2017) and Coe et al. (2019).

We target all 41 clusters with two orbits of WFC3/IR comprising observations in F105W, F125W, F140W, and F160W. Five clusters are observed with an additional pointing, for a total of 46 IR fields. We take advantage of existing archival ACS imaging, and for the 18 clusters without any F435W, F606W, and F814W we observe three orbits total, with one orbit per filter. For the ACS imaging, we also observe WFC3/IR fields in parallel, which are not explored in this work. The observations are split into two epochs separated by about a month to facilitate variability search. Twenty additional orbits were allocated for variability target of opportunity follow-up.

The SExtractor (version 2.8.6; Bertin \& Arnouts 1996) object selection and HST photometry are described by Coe et al. (2019), which we summarize here. First, we use the AstroDrizzle package (Gonzaga et al. 2012) to combine all subexposures from each filter. After aligning the filters to the same pixel frame, we correct the absolute astrometry with the Wide-field Infrared Survey Explorer point-source catalog (Wright et al. 2010). Then, we construct the final drizzled images by sampling the point-spread functions of both the ACS and WFC3/IR cameras in 30 milli-arcsecond (mas) pixel ${ }^{-1}$ and 60 mas pixel $^{-1}$ scales.

Finally, RELICS produces the full photometric catalogs of each cluster field. In this work, we use the catalogs based on a detection image comprised of the 60 mas weighted stack of all WFC3/IR imaging. The source extraction was performed with 
SExtractor in dual-image mode, with fluxes measured within the isophotal apertures. All fluxes are corrected for Galactic extinction, assuming the extinction law by Schlafly \& Finkbeiner (2011). We find nominal $5 \sigma$ depths of 27.1-27.6 $\mathrm{AB}$ mag for the ACS filters and 26.0-26.6 AB mag for the WFC3/IR filters.

\subsection{Photometric Redshifts}

In general, photometric-redshift codes use input photometric bands to identify a sharp change in flux between two wavelengths and, by leveraging with data at other wavelengths, infer the presence of the Lyman break (caused by the line-ofsight neutral intergalactic medium absorbing photons at rest wavelengths shorter than $1216 \AA$ ), the Balmer break (a feature more pronounced in older stellar populations), or strong nebular emission lines. The main difference between photometric redshifts and other selection methods, such as color and magnitude limits, is that the former is able to assign a likelihood to each redshift by fitting the observed photometry to synthetic photometry from a library of redshifted stellar population templates.

In this work, we use two independent photometric-redshift fitting codes to identify high- $z$ galaxy candidates: the Bayesian photometric-redshift code (BPZ v1.99.3; Benítez 2000; Benítez et al. 2004; Coe et al. 2006) and the Easy and Accurate Z (photometric redshifts) from Yale (EAZY; Brammer et al. 2008). Both are similar in that they fit a variety of empirically driven galaxy SEDs to the data and find the template and redshift that best represents the object. While these codes have been extensively compared for blank-field galaxies at lower redshifts $(z<6$; Brinchmann et al. 2017), in this work we explored their differences for galaxies in lensing fields at higher redshifts. Besides the choice of template SEDs, perhaps the biggest difference between BPZ and EAZY is the handling of the magnitude prior. In BPZ, the Bayesian prior $P\left(z, t \mid m_{0}\right)$ is a redshift and magnitude $\left(m_{0}\right)$-dependent prior applied to each template $t$, so as to down-weight the likelihood of, for example, the unphysical presence of bright elliptical galaxies at very high $(z>4)$ redshifts. Similarly, EAZY weights the $P(z)$ with a template-dependent $K$-band luminosity prior. In the following sections, we present our assumptions for each fitting code and use of priors.

\subsubsection{BPZ Redshifts}

BPZ compares the observed RELICS fluxes with PEGASE (Fioc \& Rocca-Volmerange 1997) SED templates. BPZ employs a Madau (1995) intergalactic medium attenuation, which accentuates the Lyman break. The default templates span a range of rest-frame $U V J$ colors, and are combined to produce SEDs with synthetic spectra similar to the high-quality spectra of most galaxies (e.g., with $\leqslant 1 \%$ outliers; see Coe et al. 2013), including red dusty starforming galaxies. We use the default BPZ templates for fitting redshifts in this work.

We found that for BPZ some simple prior must be assumed to avoid an overpopulation of quiescent-like SEDs at high redshift. With this prior, we calculate the posterior $P(z)$ for every object in each RELICS field and define our accepted BPZ redshifts as the redshift corresponding to the mode of the final probability function.
To test the reliability of our assumed prior for lensed galaxies, we conduct an input-output redshift simulation. For each BPZ template, we calculate their synthetic fluxes, apply Gaussian perturbations, and assign uncertainties based off of the average magnitude-dependent uncertainties of the RELICS data. We then redshift each template and assign a range of magnitudes, and run $\mathrm{BPZ}$ to test the recovery. We find that BPZ accurately recovers $5.5<z<8.5$ galaxies at all magnitudes $27<m<22$ with a $68 \%$ scatter in the recovery distribution of less than 0.2 from their true input redshifts. For elliptical/evolved galaxy SEDs, the recovery is less $(0.4<\sigma<0.6)$, and the outlier fraction increases with fainter elliptical galaxies. We conclude that the BPZ prior is not causing us to miss a substantial population of bright $z>5.5$ galaxies.

\subsubsection{EAZY Redshifts}

Similar to BPZ, the EAZY photometric-redshift code creates the redshift likelihood density function $(P(z))$ by computing the $\chi^{2}$ between the observed fluxes and a linear combination of redshifted empirical SED templates. EAZY includes 7 default templates from PEGASE stellar population models (Fioc \& Rocca-Volmerange 1997), a red, highly dust-obscured galaxy (Maraston 2005), and an extreme, high-equivalent width (EW) nebular emission line galaxy (Erb et al. 2010).

Similar to our test with BPZ, we explore the reliability of the default EAZY prior for lensed galaxies at high redshift. We calculated synthetic fluxes from the EAZY templates, applied magnitude-dependent flux uncertainties that are similar to the RELICS data, and redshift them to $5.5<z<8.5$. We found that the default prior has poor recovery and tends to systematically prefer the high-EW low- $z$ solutions over the correct input high- $z$ solutions. We therefore chose to adopt a flat prior for EAZY redshifts.

\subsubsection{Redshift Designation}

To construct our final sample of high- $z$ galaxy candidates, we ultimately choose to adopt the average redshift between the BPZ and EAZY estimates. We accordingly propagate the redshift uncertainties of the two codes to find the final redshift uncertainty $\sigma=0.5 \sqrt{\sigma_{\mathrm{EAZY}}^{2}+\sigma_{\mathrm{BPZ}}^{2}}$. When their redshifts differ by $\Delta z>1$, we adopt the code with the higher redshift solution and use its uncertainty. The two codes differ by a large amount for objects that have a degenerate low-redshift solution $(z \sim 1-2)$ of a red, highly dust-obscured galaxy, or one with an evolved stellar population. A slight preference to one of these solutions, due to the different assumed templates, is the primary cause for the few cases where BPZ and EAZY redshifts differ by $\Delta z>1$ (see Figure 1 ).

We note that the redshifts of EAZY and BPZ agree for the brightest high-redshift candidates in RELICS (F160W $<24.5$ AB mag). However, the two codes differ for some of the fainter candidates. Had we adopted BPZ redshifts for our selection, our samples would be $75 \%$ the size of our fiducial sample. This makes sense because as we found above, BPZ has difficulty in identifying evolved, faint galaxies at $z>5.5$. We aim to validate our sample further by a close inspection of the available Spitzer photometry and follow-up spectroscopy. In the next section, we describe the rest of our selection 

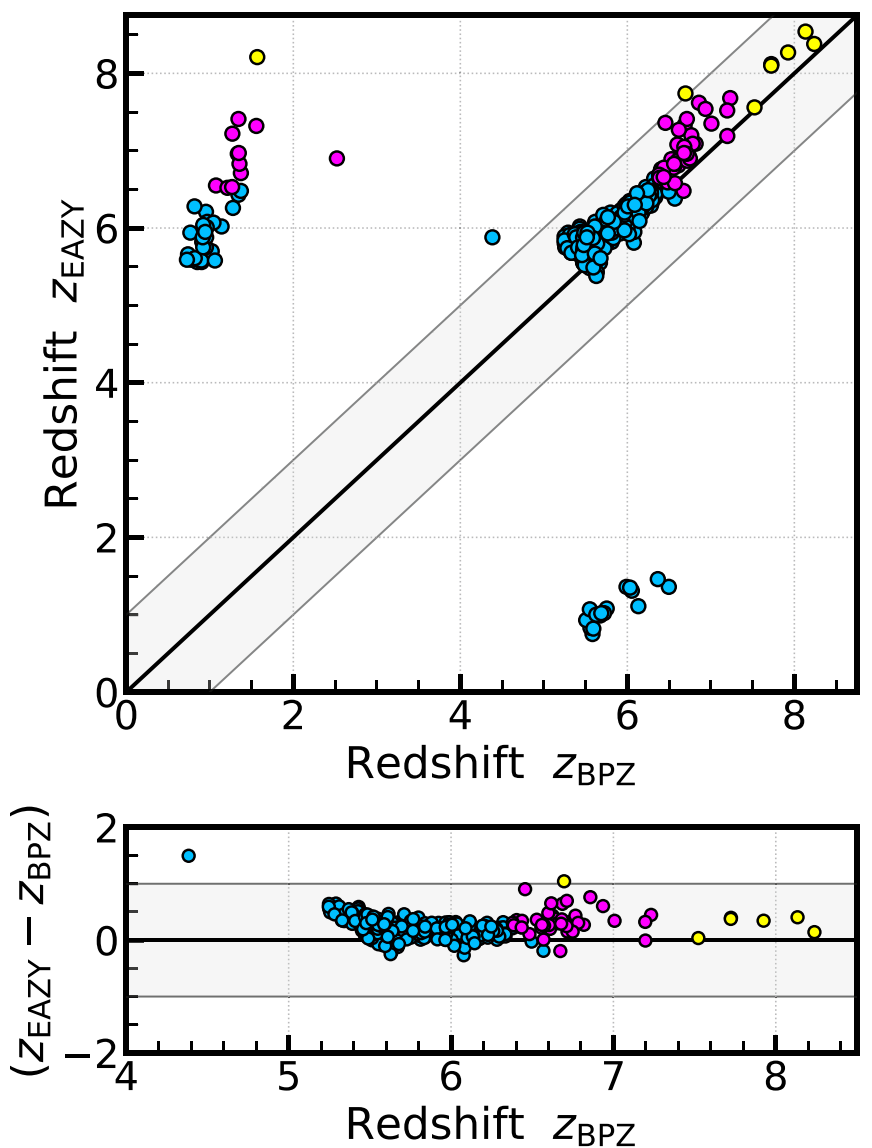

Figure 1. Comparison of EAZY and BPZ photometric redshifts for our high- $z$ sample. The gray regions show $\Delta z= \pm 1$. Blue, magenta, and yellow represent our $z \sim 6,7$, and 8 samples respectively. Objects with a high difference in redshift are due to one code preferring a $z \sim 1-2$ dusty or high EW nebular emission line galaxy. Redshifts from BPZ and EAZY closely agree for all bright $(\mathrm{F} 160 \mathrm{~W}<24.5)$ galaxies.

methods and visual inspection of each high-redshift galaxy candidate.

\subsection{High-z Sample Selection}

The RELICS catalogs contain a combined total of over 76,000 sources. From these sources, we identify 2425 objects with appreciable likelihood at $z>5.5, P(z>5.5)>40 \%$. After initial visual inspections, we found that galaxies only appeared to be bona fide candidates (that is, a signal-to-noise ratio $>3$, small sizes, and detections in individual infrared bands) if at least one of the photometric-redshift fitting codes had a median or peak likelihood at $z>5.5$. This lead us to adopting a single redshift per object to produce a complete candidate list. We took advantage of our use of two independent photometric-redshift codes by assigning the redshift of each object to be the average of the BPZ and BPZ estimates unless they differ by $\Delta z>1$, in which case we adopted the higher redshift solution. We note that the BPZ and EAZY redshifts are in approximate agreement $(|\Delta z|<1)$ for $87 \%$ of the sample, and are in excellent agreement for the brightest sources $(\mathrm{F} 160 \mathrm{~W}<25.5)$. We then selected objects with $z_{\text {phot }}>5.5$ to reduce the initial candidate list to 1337 objects. We further refined the sample by selecting galaxies

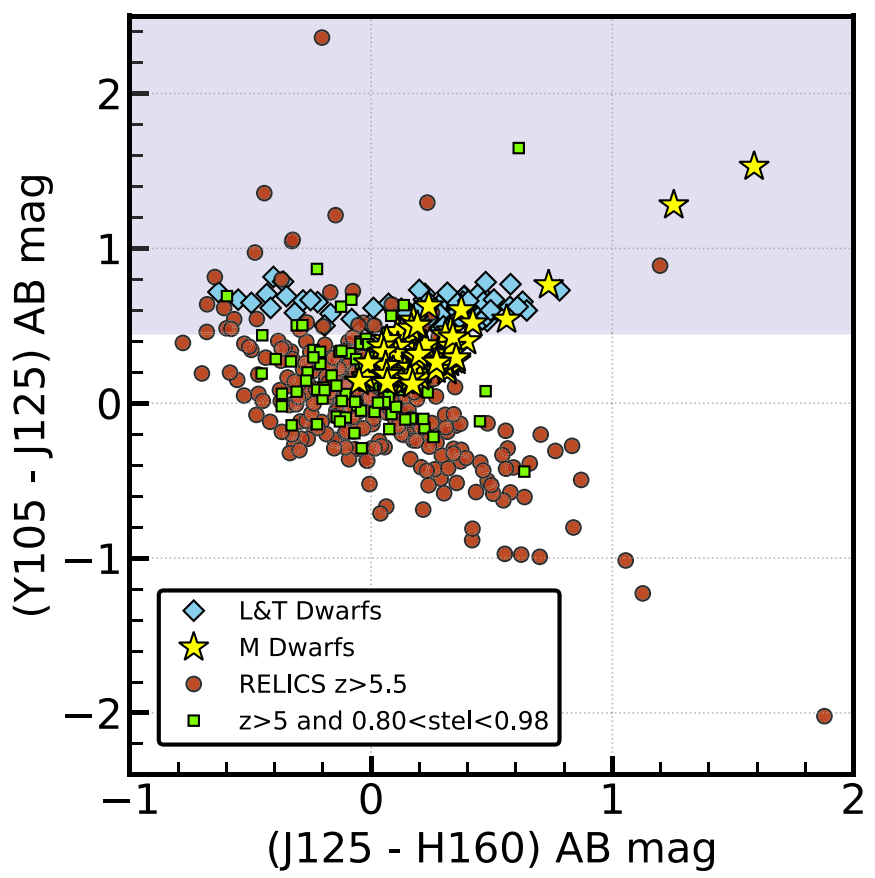

Figure 2. The YJH colors of the RELICS high- $z$ galaxy candidates. The red circles and green squares are objects with $z_{\text {phot,Max }}>5.5$, where the latter are those with high stellarity $(>0.8)$. The blue diamonds and yellow stars are colors of known L \& T and M dwarfs respectively, taken from IRTF observed spectral library (Cushing et al. 2005; Rayner et al. 2009). Most of the high-redshift galaxy candidates with high stellarity (green squares) have colors dissimilar from the dwarf stars. We remove all objects with $z_{\text {phot,Max }}>5.5,(Y-J)>$ 0.45 , and stellarity $>0.8$, which correspond to the green squares in the blue shaded region.

with an F160W detection greater than $3 \sigma$ as adopted by Bradley et al.(2014), reducing to 841 objects.

With any high- $z$ sample, we must be diligent to remove contamination by foreground Galactic stars. L-, T-, and M-dwarf stars and brown dwarfs have similar broadband IR colors as $z=6-7$ galaxies (Tilvi et al. 2013). The SExtractor "stellarity" parameter is one potentially effective way of doing so, with generally effective discriminatory power to $J<25$ or $J<26$ reported by Finkelstein et al. (2015) and Bouwens et al. (2015), respectively, but with lesser reliability fainter than these levels. This is exacerbated in lensing fields where high stellarity objects could be stars or strongly lensed high $-z$ galaxies. To avoid these objects, we use both stellarity and a color selection to reduce our stellar contamination.

First, we outright remove objects with a stellarity $\geqslant 0.98$ (111 objects) because these objects have very low FWHM $(<0$ ". 25$)$ and magnitudes systematically brighter than expected for typical lensing magnifications (mean of F160W $\sim 22.8 \mathrm{AB}$ mag, well above the distribution of magnitudes of lensed $z>6$ galaxies from CLASH; see Bradley et al. 2014). While our stellarity limit is higher than the one used by Oesch et al. (2012) on blank fields, we note that the fields used in this work contain strong gravitational lenses capable of magnifying compact galaxies with high stellarity. Such high- $z$ galaxies have been found by Bouwens et al. (2017a) with very compact light profiles and physical sizes as small as $<50 \mathrm{pc}$ (see also Vanzella et al. 2016, 2017). In addition, recent spectroscopy of some of the high stellarity, extremely bright sources in our RELICS sample (in prep) reveal the SEDs of these objects 


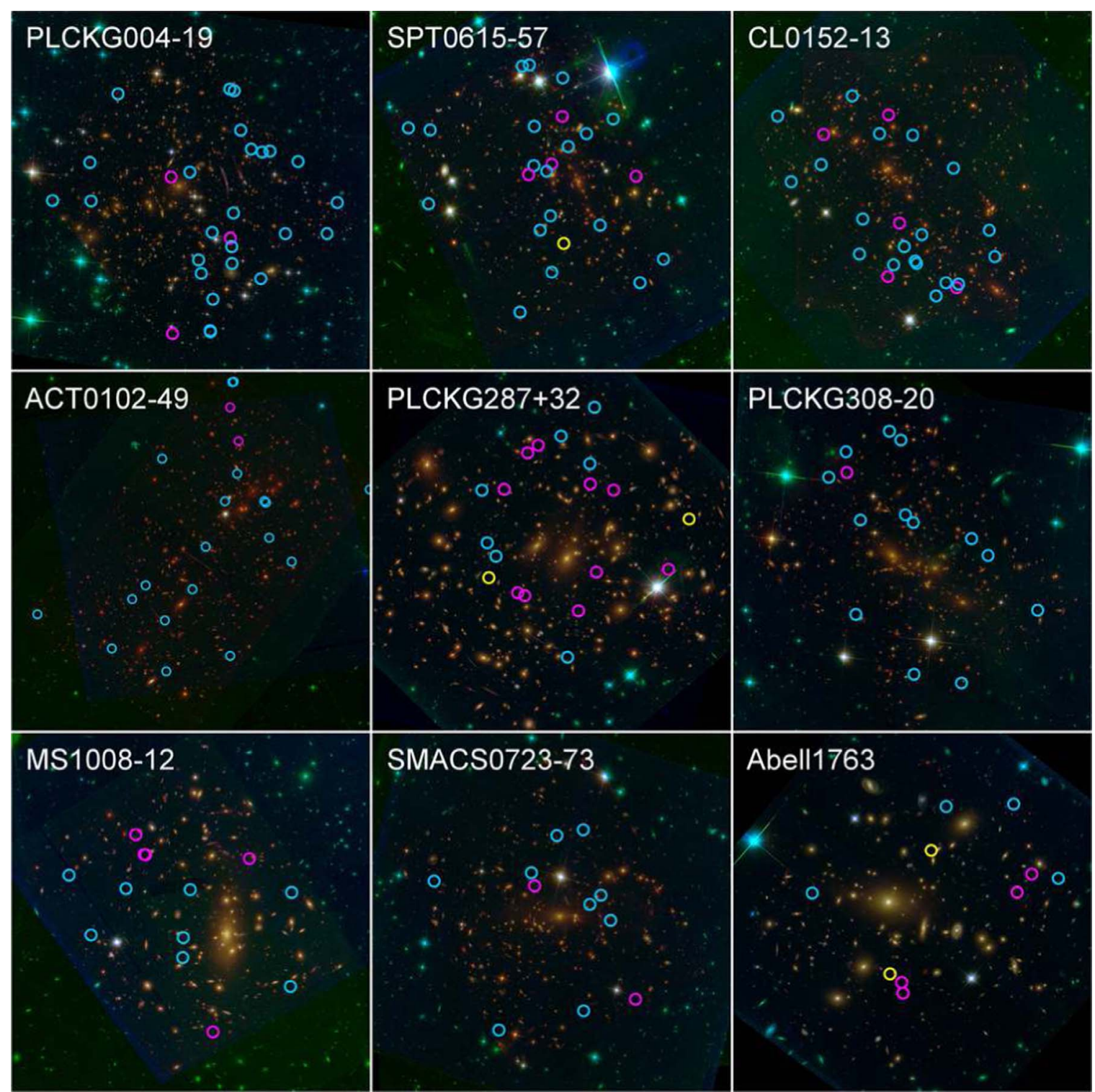

Figure 3. Color images of the top nine clusters after rank ordering by the number of $z>5.5$ galaxies they produce (excluding RXS $0603+42$ whose two WFC $3 /$ IR pointings are separated by $6^{\prime}$ ). North is up, and east is to the left. The images are scaled to $3 ! 25 \times 3 ! 25$, except for ACT $0102-49$ which is $4 ! 25 \times 4 ! 25$. The cyan, magenta, and yellow circles mark the location of the $z \sim 6,7$, and 8 candidate galaxies, respectively.

better match starburst galaxies than known low-mass dwarf stars.

Second, we compare the $Y J H$ colors of the RELICS high- $z$ sample with the colors of known L-, T-, and M-dwarf stars (Cushing et al. 2005; Rayner et al. 2009). Figure 2 shows that the colors of most objects at intermediate stellarity (stellarity of $0.80-0.98$ ) are dissimilar to the colors of known L- and T-dwarf stars. Nevertheless, we make a selection to omit high- $z$ candidates that satisfy both intermediate stellarity $(80 \%-98 \%)$ and $(Y-J)>0.45$ colors, which removes 11 high- $z$ candidates from our sample. We also note that there are on order $\approx 10$ candidates with both intermediate stellarity and colors close to most $\mathrm{M}$ dwarfs, but the $Y J H$ colors or other available color combinations do not well distinguish $\mathrm{M}$ dwarfs from high- $z$ galaxies. We estimate that there may be an M-dwarf contamination rate of $\sim 5 \%-10 \%$.
Finally, we conduct an extensive visual inspection of all remaining candidates to ensure no spurious artifacts entered the sample. The most common candidate that was removed by visual inspection was stellar diffraction spikes that were segmented into their own object by SExtractor. This is because the WFC3/IR diffraction spikes do not overlap with the ACS diffraction spikes, and thus they can appear as optical dropouts if SExtractor identified part of the spike as an individual object. The SEDs of these spikes are flat in $f_{\nu}$ with a strong spectral break, very similar to a high- $z$ galaxy. Other removed candidates included some that appeared very large in size (isophotal area $>100$ pixels) and spherical in shape (not obviously stretched in one direction by lensing shear), indicative of foreground objects. These objects also had a significant low- $z$ solution $P(z<4)>40 \%$. Other removed objects were within the faint diffuse edges of a foreground 
Table 1

High- $z$ Number Counts Per Cluster

\begin{tabular}{|c|c|c|c|c|c|c|c|c|}
\hline Cluster & $\begin{array}{l}\text { R.A. } \\
\text { (J2000) }\end{array}$ & $\begin{array}{c}\text { Decl. } \\
\text { (J2000) }\end{array}$ & $\begin{array}{c}\text { Cluster } \\
\text { Redshift }\end{array}$ & $\begin{array}{c}\text { Planck Mass } \\
\left(10^{14} M_{\odot}\right)\end{array}$ & $N_{\text {total }}$ & $N_{z=6}$ & $\mathrm{~N}_{z=7}$ & $N_{z=8}$ \\
\hline PLCK G004-19 & $19: 17: 4.50$ & $-33: 31: 28.5$ & 0.540 & 10.36 & 28 & 25 & 3 & 0 \\
\hline SPT 0615-57 & $06: 15: 54.2$ & $-57: 46: 57.9$ & 0.972 & 6.77 & 24 & 19 & 4 & 1 \\
\hline CL 0152-13 & 01:52:42.9 & $-13: 57: 31.0$ & 0.833 & $\ldots$ & 24 & 19 & 5 & 0 \\
\hline АCT 0102-49 & 01:02:53.1 & $-49: 14: 52.8$ & 0.870 & 10.75 & 21 & 19 & 2 & 0 \\
\hline PLCKG 287+32 & $11: 50: 50.8$ & $-28: 04: 52.2$ & 0.39 & 14.69 & 19 & 7 & 10 & 2 \\
\hline PLCKG 308-20 & $15: 18: 49.9$ & $-81: 30: 33.6$ & 0.480 & 10.32 & 14 & 13 & 1 & 0 \\
\hline MS 1008-12 & $10: 10: 33.6$ & $-12: 39: 43.0$ & 0.306 & 4.94 & 13 & 8 & 5 & 0 \\
\hline RXS $0603+42$ & $06: 03: 12.2$ & $+42: 15: 24.7$ & 0.228 & 10.76 & 13 & 13 & 0 & 0 \\
\hline SMACS 0723-73 & $07: 23: 19.5$ & $-73: 27: 15.6$ & 0.390 & 8.39 & 11 & 9 & 2 & 0 \\
\hline A 1763 & $13: 35: 18.9$ & $+40: 59: 57.2$ & 0.228 & 8.13 & 10 & 4 & 4 & 2 \\
\hline MACS 0553-33 & $05: 53: 23.1$ & $-33: 42: 29.9$ & 0.430 & 8.77 & 9 & 5 & 3 & 1 \\
\hline MACS 0257-23 & $02: 57: 10.2$ & $-23: 26: 11.8$ & 0.505 & 6.22 & 9 & 8 & 1 & 0 \\
\hline RXC 0600-20 & 06:00:09.8 & $-20: 08: 08.9$ & 0.460 & 10.73 & 8 & 7 & 1 & 0 \\
\hline MACS 0025-12 & $00: 25: 30.3$ & $-12: 22: 48.1$ & 0.586 & & 7 & 6 & 1 & 0 \\
\hline A 2163 & $16: 15: 48.3$ & $-06: 07: 36.7$ & 0.203 & 16.12 & 7 & 6 & 1 & 0 \\
\hline A 1758 & $13: 32: 39.0$ & $+50: 33: 41.8$ & 0.280 & 8.22 & 6 & 6 & 0 & 0 \\
\hline $\mathrm{RXC} 0018+16$ & $00: 18: 32.6$ & $+16: 26: 08.4$ & 0.546 & 9.79 & 6 & 6 & 0 & 0 \\
\hline A 520 & 04:54:19.0 & $+02: 56: 49.0$ & 0.203 & 7.80 & 6 & 5 & 1 & 0 \\
\hline MACS $0308+26$ & 03:08:55.7 & $+26: 45: 36.8$ & 0.356 & 10.76 & 6 & 6 & 0 & 0 \\
\hline RXC 0911+17 & 09:11:11.4 & $+17: 46: 33.5$ & 0.505 & 6.99 & 6 & 4 & 1 & 1 \\
\hline A 295 & $02: 45: 31.4$ & $-53: 02: 24.9$ & 0.300 & 6.78 & 6 & 4 & 1 & 1 \\
\hline A 665 & 08:30:57.4 & $+65: 50: 31.0$ & 0.182 & 8.86 & 5 & 5 & 0 & 0 \\
\hline A 3192 & 03:58:53.1 & $-29: 55: 44.8$ & 0.425 & 7.20 & 5 & 2 & 3 & 0 \\
\hline PLCKG 209+10 & $07: 22: 23.0$ & $+07: 24: 30.0$ & 0.677 & 10.73 & 5 & 5 & 0 & 0 \\
\hline A 2537 & 23:08:22.2 & $-02: 11: 32.4$ & 0.297 & 5.52 & 5 & 5 & 0 & 0 \\
\hline SPT 0254-58 & $02: 54: 16.0$ & $-58: 57: 11.0$ & 0.438 & 9.69 & 4 & 3 & 1 & 0 \\
\hline $\mathrm{RXC} 0142+44$ & $01: 42: 55.2$ & $+44: 38: 04.3$ & 0.341 & 9.02 & 4 & 3 & 1 & 0 \\
\hline A 1300 & 11:31:54.1 & $-19: 55: 23.4$ & 0.307 & 8.97 & 4 & 2 & 2 & 0 \\
\hline MACS 0159-08 & 01:59:49.4 & $-08: 50: 00.0$ & 0.405 & 7.20 & 4 & 3 & 1 & 0 \\
\hline MACS 0035-20 & $00: 35: 27.0$ & $-20: 15: 40.3$ & 0.352 & 7.01 & 4 & 3 & 1 & 0 \\
\hline WHL 0137-08 & $01: 37: 25.0$ & $-08: 27: 25.0$ & 0.566 & 8.93 & 4 & 4 & 0 & 0 \\
\hline А 697 & $08: 42: 58.9$ & $+36: 21: 51.1$ & 0.282 & 11.0 & 4 & 4 & 0 & 0 \\
\hline PLCKG 138-10 & $02: 27: 06.6$ & $+49: 00: 29.9$ & 0.702 & 9.48 & 3 & 2 & 1 & 0 \\
\hline PLCKG 171-40 & $03: 12: 56.9$ & $+08: 22: 19.2$ & 0.270 & 10.71 & 3 & 3 & 0 & 0 \\
\hline RXC $0032+18$ & $00: 32: 11.0$ & $+18: 07: 49.0$ & 0.396 & 7.61 & 3 & 2 & 1 & 0 \\
\hline RXC 0232-44 & $02: 32: 18.1$ & $-44: 20: 44.9$ & 0.284 & 7.54 & 3 & 3 & 0 & 0 \\
\hline RXC $0949+17$ & 09:49:50.9 & $+17: 07: 15.3$ & 0.383 & 8.24 & 3 & 3 & 0 & 0 \\
\hline RXC 1514-15 & $15: 15: 00.7$ & $-15: 22: 46.7$ & 0.223 & 8.86 & 2 & 2 & 0 & 0 \\
\hline RXC 2211-03 & $22: 11: 45.9$ & $-03: 49: 44.7$ & 0.397 & 10.5 & 2 & 2 & 0 & 0 \\
\hline A 2813 & $00: 43: 25.1$ & $-20: 37: 14.8$ & 0.292 & 8.13 & 2 & 2 & 0 & 0 \\
\hline MACS0417-11 & $04: 17: 33.7$ & $-11: 54: 22.6$ & 0.443 & 12.25 & 0 & 0 & 0 & 0 \\
\hline
\end{tabular}

elliptical galaxy, a result of SExtractor mistakenly identifying the noisy edge of a foreground galaxy as its own object. Similarly, sometimes the object was clearly an oversegmented part of another galaxy that SExtractor identified as its own object. Finally, the last class of removed objects were those that were visually identified as a noise artifact: all their signal was coming from a few pixels, typically with an isophotal area $<15$ pixels. In summary we discover 257 candidate galaxies at $z \sim 6,57$ at $\sim 7$ and 8 at $z \sim 8$, for 322 galaxies in total.

\section{Results}

Figure 3 shows RGB color images of the top nine high- $z$ producing fields (excluding RXS 0603+42, whose two WFC3/IR pointings are separated by $6^{\prime}$ ), and the overlaid position of each high- $z$ candidate. These images show that our candidate high- $z$ galaxies are not clustered around the edges of the IR detector, thanks to our visual screening of every candidate. Cerny et al. (2017) conducted an analysis of the first five RELICS clusters, which span the range of masses and redshifts of the clusters in the full program. They found that these five clusters had lensing efficiencies of similar strength to the Frontier Fields. In future works, we will publish the lens models and magnifications of all 41 clusters, which will allow us to explore the lensed counter images.

The breakdown of the number of galaxies in each cluster and each redshift bin are shown in Table 1. Figure 4 displays these number counts per cluster as a histogram. Clearly, some clusters produce many more high- $z$ candidates than others, even after accounting for the five clusters that have additional WFC3/IR pointings. For example, the top $\approx 12 \%$ of the high- 

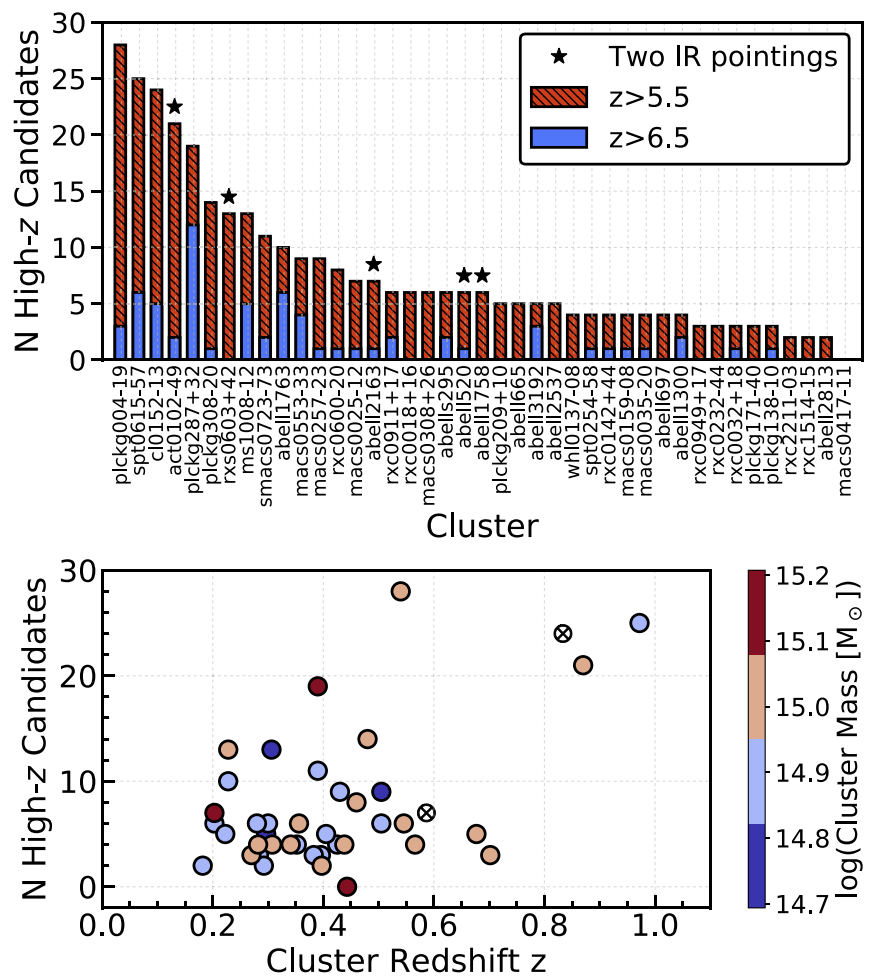

Figure 4. Number of high- $z$ candidates for each cluster field observed by RELICS. Top: histograms of the number of $z>5.5$ candidate galaxies (solid blue and hatched red) and number of $z>6.5$ candidates (solid blue only). The five clusters with two WFC3/IR pointings are noted with the solid stars. Some clusters produce considerable numbers of high- $z$ candidates compared with others. Bottom: the number of high- $z$ candidates per cluster as a function of the cluster redshift. The blue-tored colors portray the cluster mass $\left(\mathrm{M}_{500}\right)$ from Planck Collaboration et al. (2016), where the crossed circles show the two clusters without mass estimates. There is a weak correlation between the cluster redshift and the ability of a cluster to lens high- $z$ sources, and little correlation with the cluster mass.

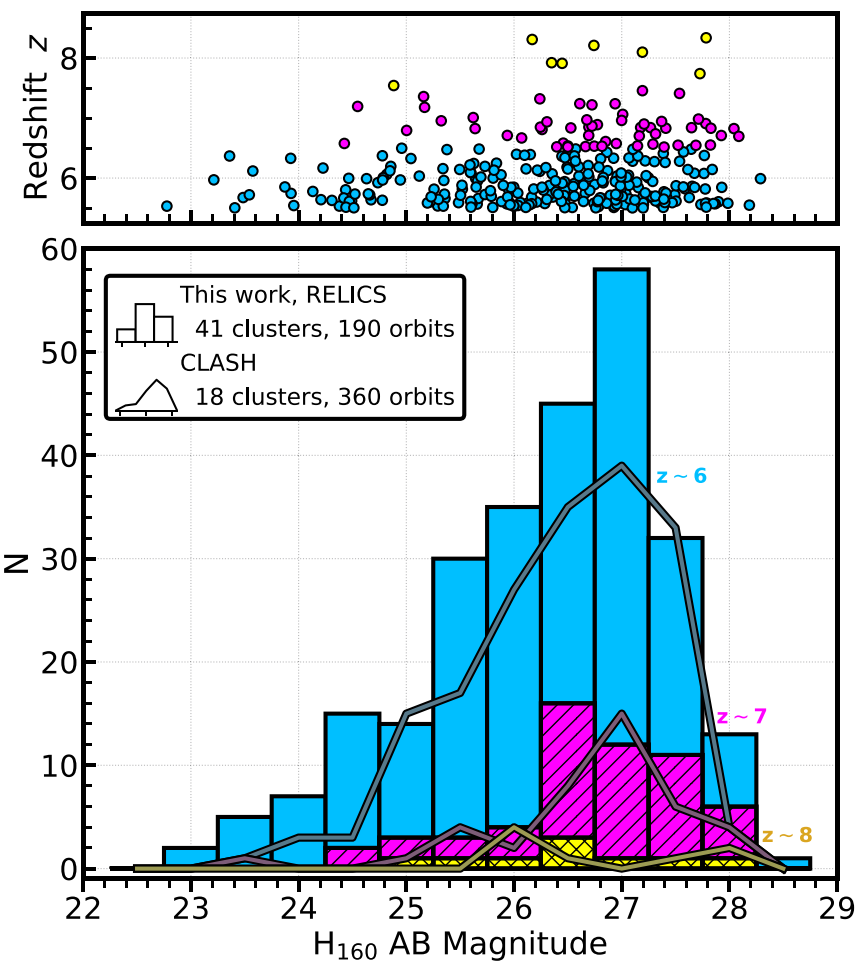

Figure 5. Distribution of WFC3 F160W $H$-band observed (lensed) magnitude for our $z \sim 6$, 7, and 8-10 RELICS samples in solid blue, left-hatched magenta, and right-hatched yellow histograms. The curves show the distribution of $z \sim 6,7$, and $8-10$ galaxies (blue, magenta, and yellow, respectively) from the first 18 clusters and 360 orbits of CLASH (Bradley et al. 2014). The above panel shows the photometric redshifts of individual RELICS galaxies as a function of their magnitude. RELICS produces a similar magnitude distribution of high- $z$ galaxies as CLASH. 


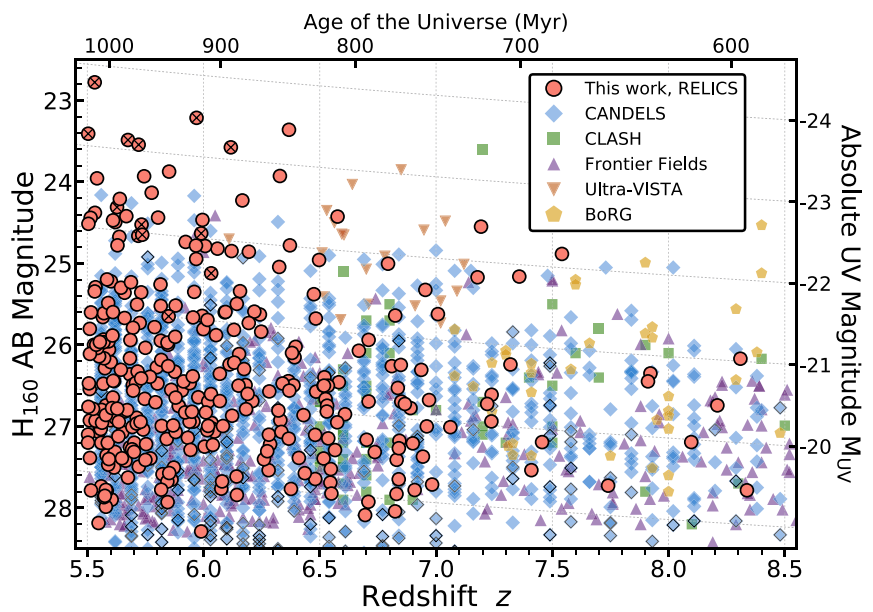

Figure 6. $H$-band magnitude as a function of redshift. The high- $z$ galaxy candidates from RELICS are shown as salmon-colored circles. Circles filled with an " $X$ " mark candidates from the cluster field RXS $0603+42$, which is at a low Galactic latitude $\left(b \approx 10^{\circ}\right)$ and therefore has a potentially higher stellar contamination. The green squares are galaxies from CLASH (Bradley et al. 2014), the purple upward triangles from the Frontier Field (Ishigaki et al. 2018), the red downward triangle from UltraVISTA (Bowler et al. 2017), the blue diamonds from CANDELS (Bouwens et al. 2015) (outlined diamonds are from the HUDF; see also Finkelstein et al. 2015), and the orange pentagons from BoRG/HIPPIES (Bradley et al. 2012; Schmidt et al. 2014; Calvi et al. 2016). Gray background lines follow the conversion from apparent magnitude to absolute UV magnitude. RELICS finds some of the brightest known galaxies at a given redshift over $z \sim 8$ to $z \sim 6$.

$z$ producing clusters contain as many candidates (almost a third of the entire $z>5.5$ sample) as the bottom $50 \%$ of the clusters. Figure 4 also shows that there appears to be little to no correlation between the ability of a cluster to produce high$z$ sources and its cluster mass, at least at these high masses. However, a weak correlation exists with the number of high- $z$ candidates and the cluster redshift. However, there may be several reasons for why some clusters are better at producing high- $z$ sources than others, such as a dependence on the cluster mass distribution and concentration, the latter of which is expected to correlate with dark matter halo mass (Bullock et al. 2001; Neto et al. 2007; Umetsu et al. 2014). In addition, there may be sample variance in the alignment of background galaxies. A comprehensive exploration of cluster-lensing strength will require a comparison between the lens models from all RELICS clusters.

\subsection{Magnitude Distribution}

Figure 5 shows distribution of F160W $H$-band magnitude for the RELICS high-redshift galaxies compared with that of the CLASH survey. In a given redshift and magnitude bin at relatively bright magnitudes $(<25 \mathrm{AB}$ mag), RELICS and CLASH yield similar number densities of high-redshift galaxies. At fainter magnitudes, RELICS begins to suffer from incompleteness and photometric-redshift uncertainties (to be quantified in future work). Each RELICS WFC3/IR field consisted of 2 orbits and yielded 7 high-redshift candidates on average. Each CLASH WFC3/IR field was deeper, consisting of six orbits and yielding 14.5 high-redshift candidates on average (Bradley et al. 2014). Where CLASH had broader science goals requiring more orbits overall (524), RELICS was optimized to efficiently yield a large number of high-redshift candidates in fewer orbits (190). We note that our total high-redshift count is inflated by $\sim 33 \%$ with respect to CLASH, given that we included candidates with high redshifts between the average of BPZ and EAZY (see Section 2.2.3). Figure 5 also highlights the abundance of bright $m_{\mathrm{AB}}<26$ candidates at a given redshift, which presents a promising sample for follow-up spectroscopy.

Figure 6 shows the $H$-band magnitude of the RELICS high- $z$ candidates as a function of redshift compared with several large surveys including CANDELS (Bouwens et al. 2015; Finkelstein et al. 2015), the HFF (Ishigaki et al. 2018), CLASH (Bradley et al. 2014), UltraVISTA (Bowler et al. 2017), and BoRG/HIPPIES (Bradley et al. 2012; Schmidt et al. 2014; Calvi et al. 2016). RELICS produces galaxies that are among the brightest at a given redshift over $z \sim 5.5-z \sim 7.5$, comparable to these much wider and deeper programs. We highlight this comparison to emphasize the efficiency of targeting strong lensing fields to produce high- $z$ candidates, which is especially relevant as the costly overheads of JWST make the telescope more efficient at smaller area surveys.

Finally, Figure 7 displays the number density of galaxies in bins of magnitude over $z=6-8$. We assume an area of $4.5 \operatorname{arcmin}^{2}$ for each of the $46 \mathrm{WFC} 3 / \mathrm{IR}$ pointings for a total survey area of $207 \mathrm{arcmin}^{2}$. The actual area will change after lens models determine the magnification maps and the effective area covered for each cluster, although typical areas from CLASH range between 4.3 and $4.8 \mathrm{arcmin}^{2}$ per cluster field. Nevertheless, we observe a clear excess in number density at the $z \sim 6$ and 7 bright magnitudes compared with unlensed fields. We note that the drop off at fainter magnitudes $\left(m_{\mathrm{AB}}>26.5\right)$ is due to survey incompleteness.

To provide a baseline comparison for our results, we make use of a LF with a double power-law fit to a comprehensive set of $z \sim 6,7$, and 8 results from the literature. A double power law has been found to work well in representing the extreme bright-end shape of the UV LF (Bowler et al. 2014, 2015; Stefanon et al. 2017; Ono et al. 2018). By comparing the bright end of our results from RELICS against that of a double-law $\mathrm{LF}$, we aim to show that the number density boost from lensing we observe exceeds even the expectation from the double-law LF, which already exhibits a much larger number of sources than a LF with a Schechter (Schechter 1976) form. As a second baseline comparison for our results, we also show the 

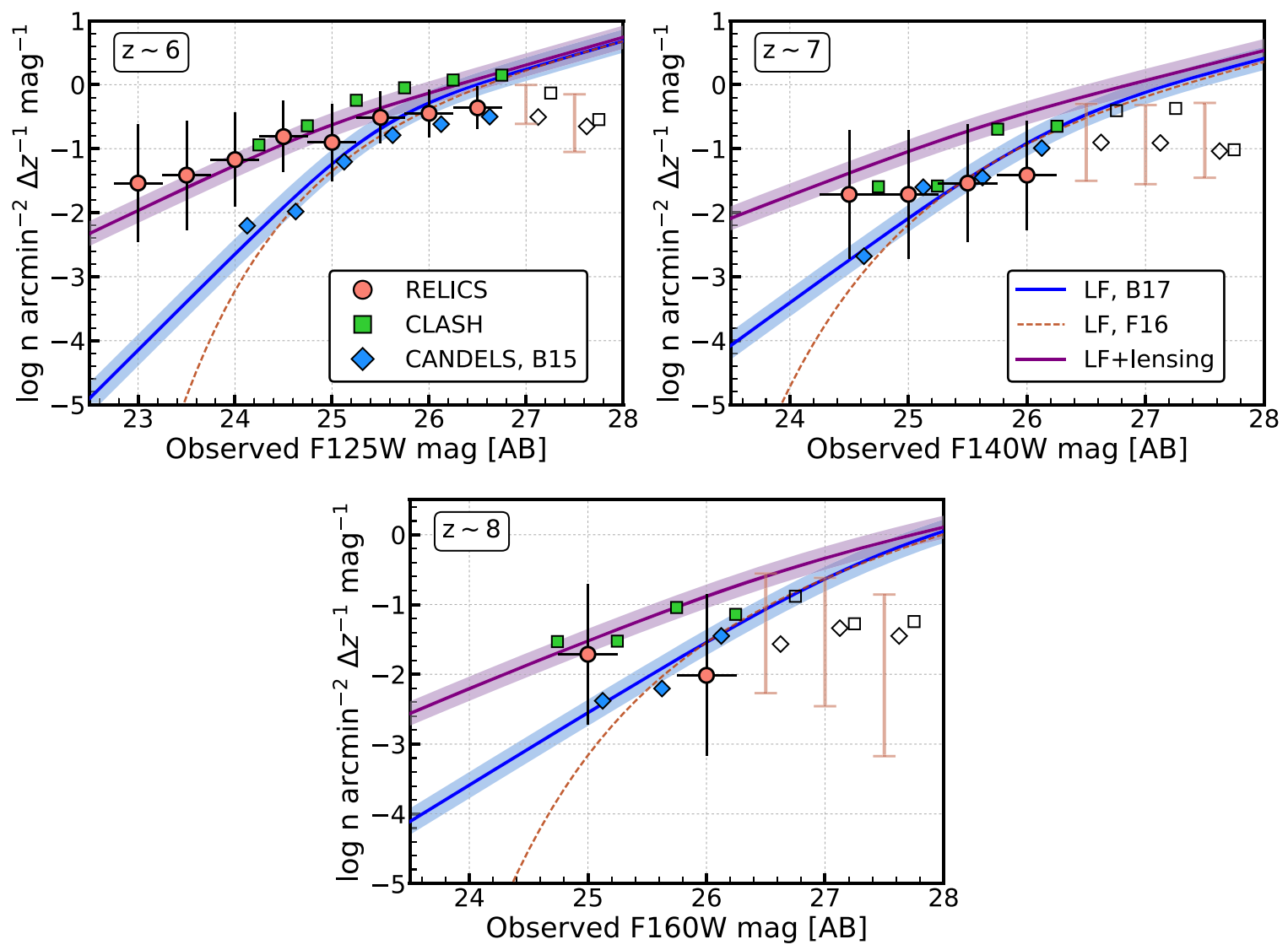

Figure 7. Number density of galaxies per magnitude and redshift bin at $z \sim 6,7$, and 8 . The observed number densities in the 41-cluster RELICS samples are shown as salmon-colored circles, with their $1 \sigma$ Poissonian uncertainties. The green squares are the observed number densities seen in CLASH by Bradley et al. (2014) and the blue diamonds are those seen in CANDELS by Bouwens et al. (2015). The blue and red-dashed curves represent the double power-law and Schechter fits to a suite of published luminosity function results (Bradley et al. 2012; Oesch et al. 2012; McLure et al. 2013; Bouwens et al. 2015; Finkelstein et al. 2015; Stefanon et al. 2017; Ono et al. 2018) from the literature (see Finkelstein 2016). The faint, capped error bars and open symbols show the number densities where faint-magnitude incompleteness begins to dominate. The purple curves represent the expected number densities from CLASH after simulating lensing effects on the Bouwens et al. (2015) literature luminosity function. Compared with CLASH, RELICS yields similar number densities of $z \sim 6$ galaxies, extending to brighter lensed magnitudes ( $\left.H m_{\mathrm{AB}}<26\right)$. At $z \sim 7$ and 8, RELICS yields somewhat lower number densities.

expectations from the literature-averaged Schechter function results from Finkelstein (2016), which features fewer sources at the extreme bright end due the bright-end shape of the Schechter function, but overall is very similar at luminosities less than $2 L^{*}$.

Next, we apply typical CLASH cluster-lensing magnifications to model how the number densities of the true LF appear under the effects of cluster lensing. We only show the effects of lensing on the double-law LF, but we note that forms of the LF produce a similar lensed shape. Comparing the lensed LF with the binned number densities of RELICS and CLASH, we find they agree with the prediction well at $z \sim 6$, and that both surveys tend to underproduce from the expected number of sources at $z \sim 7$ and 8 . It is hard to speculate if this is due to an actual bright-end decline in number density at higher redshifts, as seen by Bowler et al. (2017), until we conduct full completeness simulations and lens modeling of all 41 RELICS clusters and consider the effects of cosmic variance.

\subsection{Exceptionally Bright Sources}

The RGB image stamps of the brightest 40 galaxy candidates from RELICS for $z \sim 6$ and 7, and all candidates for $z \sim 8$ are shown in Figures 8, 9, and 10, respectively. In particular, we note the third brightest $z \sim 6$ candidate, MACS 0308-904, which has been clearly arced by the effects of lensing and is likely highly magnified. Not all highly magnified galaxies will also be arced, which makes it very difficult with the current data to distinguish between stellar contaminants and high- $z$ galaxies among the brightest candidates. We have already attempted to remove stellar contaminants by a combination of $Y J H$ colors and stellarity (Figure 2 and Section 2.3). In addition, we checked the brightest candidates in our samples with the Galactic latitude of their cluster field. We find that $\approx 6$ of our brightest $z \sim 6$ candidates come from a cluster field with relatively low Galactic latitude (RXS $0603+42$, at $b=9^{\circ} .7$ ). We specially note these objects in Figure 6 and their images and SEDs can be inspected in Figures 8 and 11. Besides this one cluster, we see no correlation between the number or brightness of high $-z$ candidates and the Galactic latitude of the cluster fields. We anticipate a deeper exploration of contaminants in the future using lensing magnifications, Spitzer photometry, and/or spectroscopic redshifts.

Following the same plotting grid of the image stamp figures, the SEDs of the brightest candidates and their photometricredshift template fits for BPZ and EAZY are shown in 


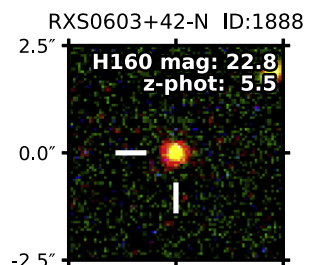

RXS0603+42-S ID:6059

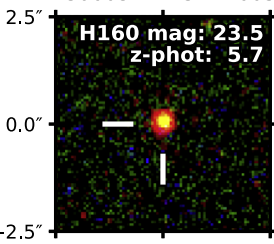

SMACS0723-73 ID:711
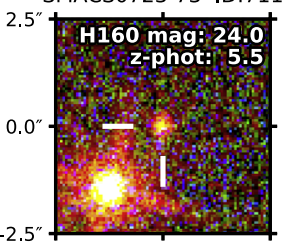

CL0152-13 ID:1508
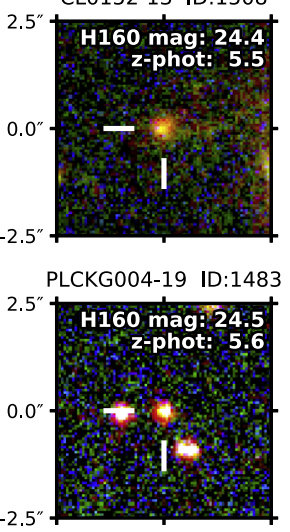

RXS0603+42-N ID:7437

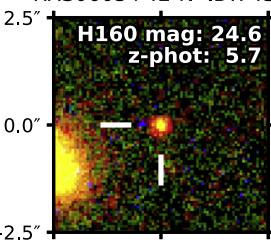

MACS0308+26 ID:438
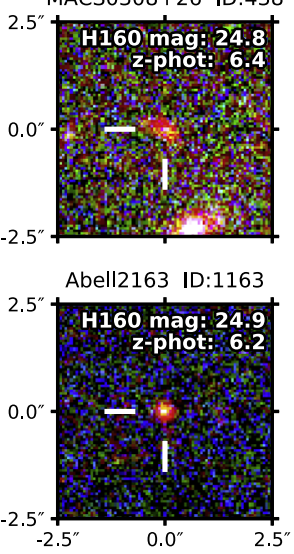

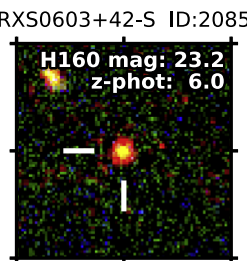

RXS0603+42-N ID:1050

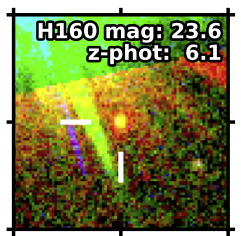

PLCKG004-19 ID:222

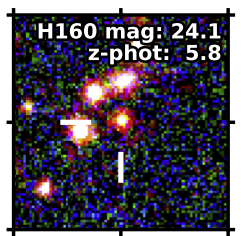

MACS0553-33 ID:1323

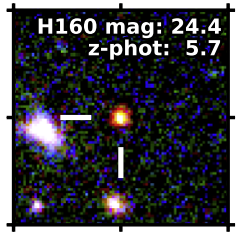

Abell2163 ID:1204

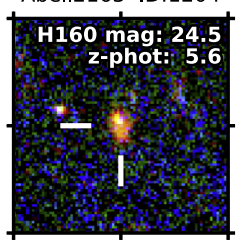

RXS0603+42-N ID:4419

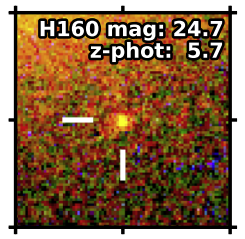

SPT0615-57 ID:325
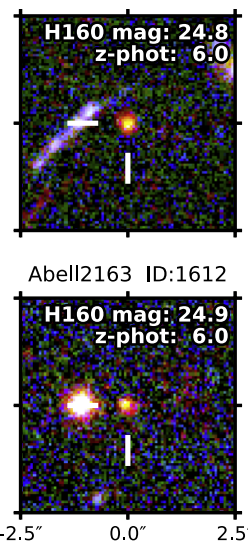

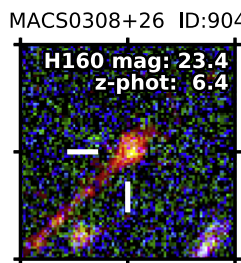

SMACS0723-73 ID:1366

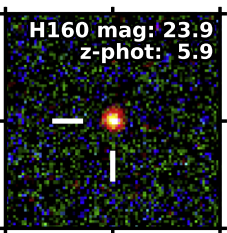

SPT0615-57 ID:261
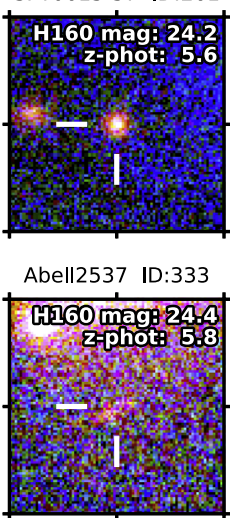

SMACS0723-73 ID:1430
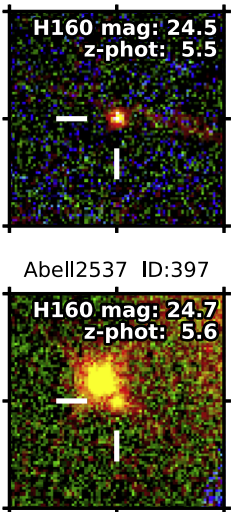

PLCKG209+10 ID:202

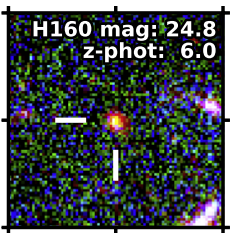

PLCKG287+32 ID:3078

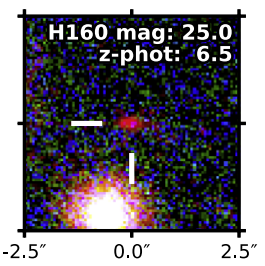

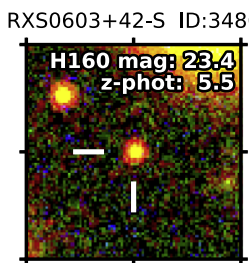

RXC0600-20 ID:840

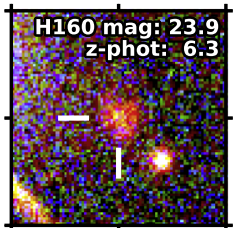

RXC2211-03 ID:196

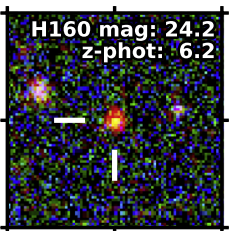

RXC0142+44 ID:323

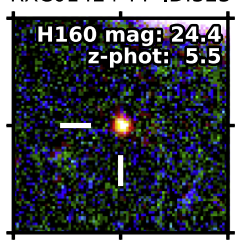

RXS0603+42-N ID:1925

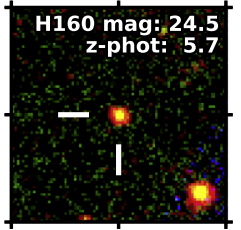

RXC1514-15 ID:550

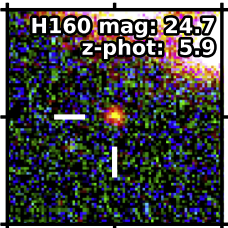

Abell1758 ID:1942
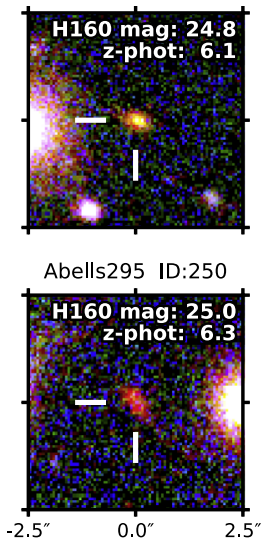

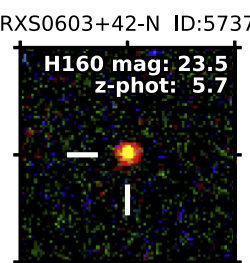

PLCKG171-40 ID:130

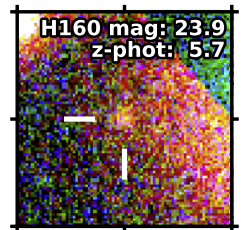

RXS0603+42-N ID:6150

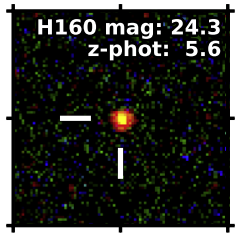

PLCKG004-19 ID:2691

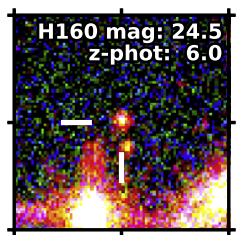

RXS0603+42-N ID:4609

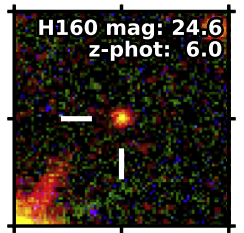

MACS0308+26 ID:249

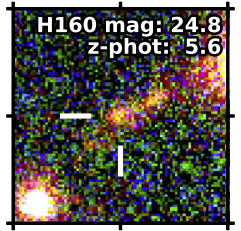

RXC0600-20 ID:1304

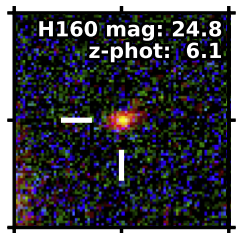

RXS0603+42-S ID:5318

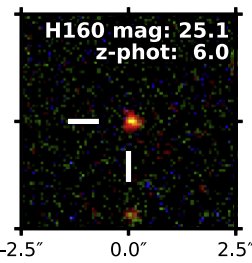

Figure 8. Brightest 40 galaxy candidates from the $z \sim 6$ RELICS sample. Each RGB color image stamp is $5^{\prime \prime} \times 5^{\prime \prime}$ with the red channel as the sum of all IR bands, the $\mathrm{G}$ channel as the ACS F814W band, and the B channel the sum of ACS F435W and F606W. The F160W H-band AB magnitude is shown within each stamp, along with the adopted redshift (see Section 2.3). The cluster name and catalog ID are shown at the top of each stamp. 


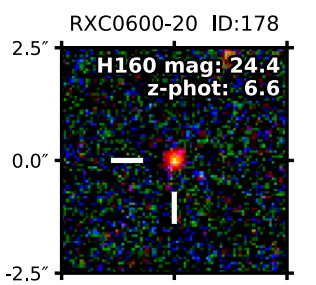

Abell3192 ID:1076

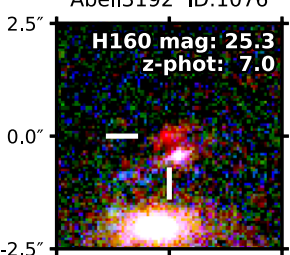

PLCKG287+32 ID:792

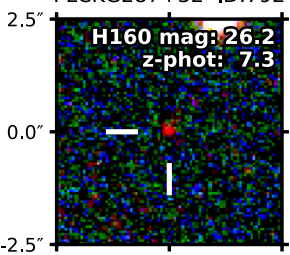

MS1008-12 ID:313

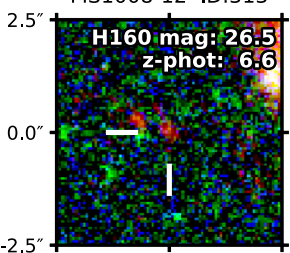

Abell520 ID:1342

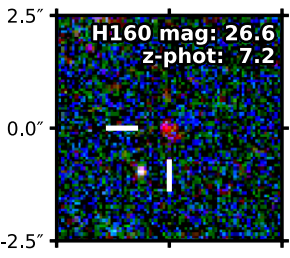

MS1008-12 ID:227

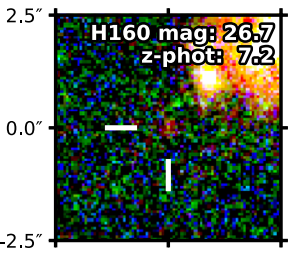

PLCKG287+32 ID:221

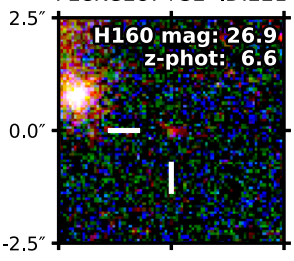

MACS0553-33 ID:463
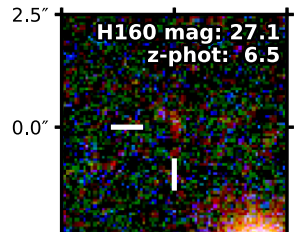

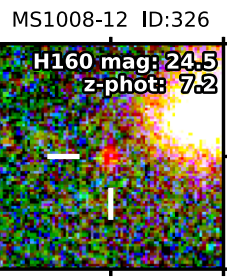

Abell1300 ID:226
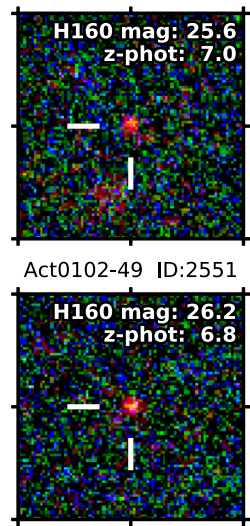

Abell1763 ID:1500

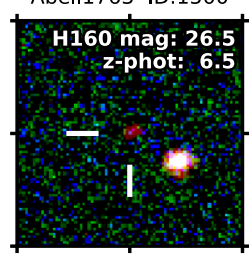

CL0152-13 ID:410

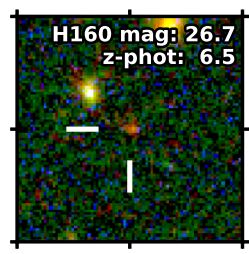

PLCKG004-19 ID:2704

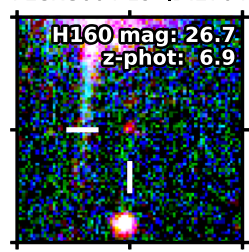

MACS0257-23 ID:787

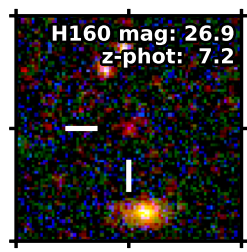

MACS0553-33 ID:865

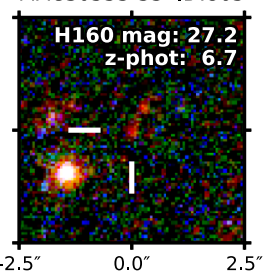

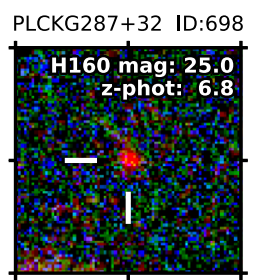

RXC0911+17 ID:419
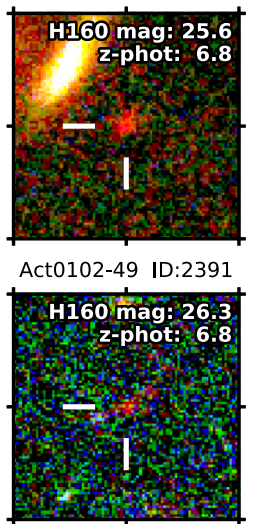

SPT0254-58 ID:476

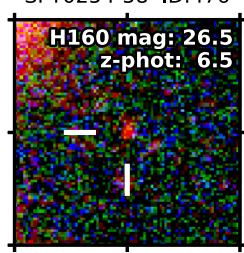

RXC0142+44 ID:1088

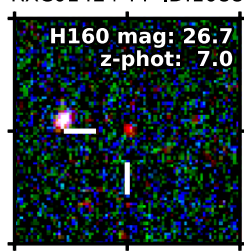

PLCKG004-19 ID:1983

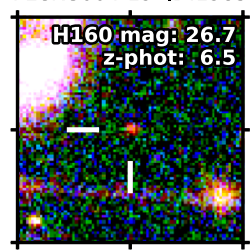

SPT0615-57 ID:548

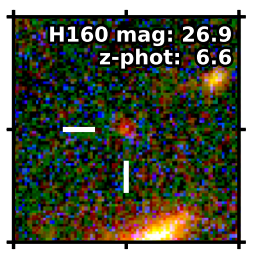

Abell1763 ID:1564

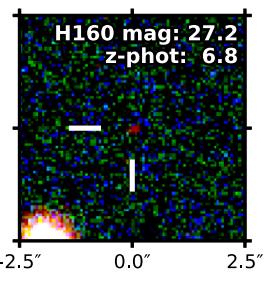

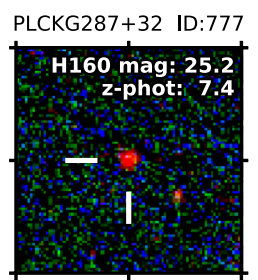

Abell1300 ID:534

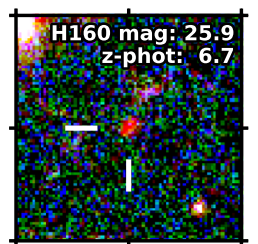

MS1008-12 ID:312

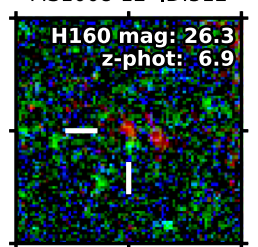

RXC0032+18 ID:571

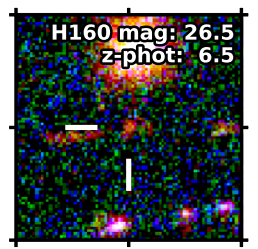

PLCKG287+32 ID:2457

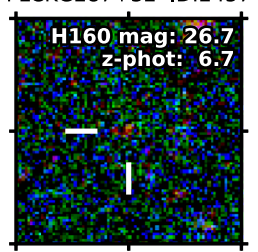

Abell2163 ID:1715

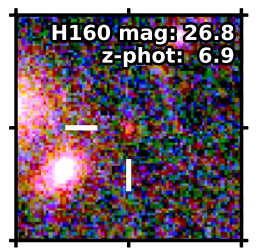

PLCKG287+32 ID:1887

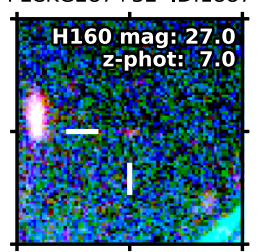

Abell1763 ID:817

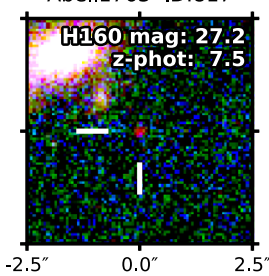

PLCKG287+32 ID:2235

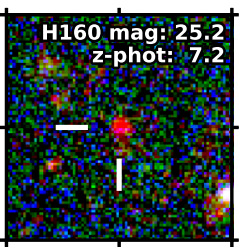

Abell3192 ID:33

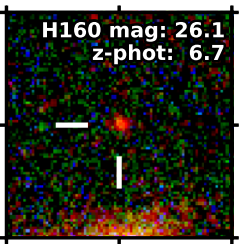

PLCKG287+32 ID:2252

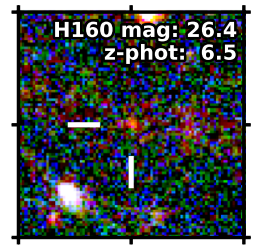

SMACS0723-73 ID:1317

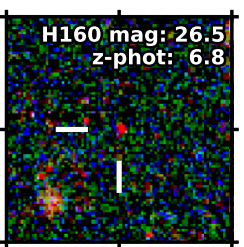

CL0152-13 ID:259

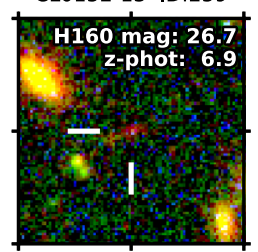

SMACS0723-73 ID.599

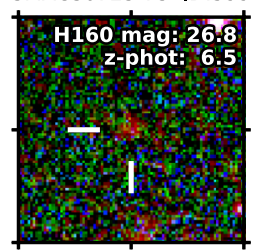

PLCKG004-19 ID:1156

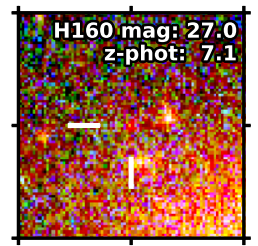

PLCKG287+32 ID:2199

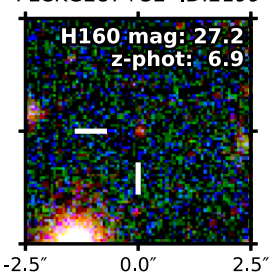

Figure 9. Same as Figure 8, but for the brightest 40 objects from the $z \sim 7$ sample. 


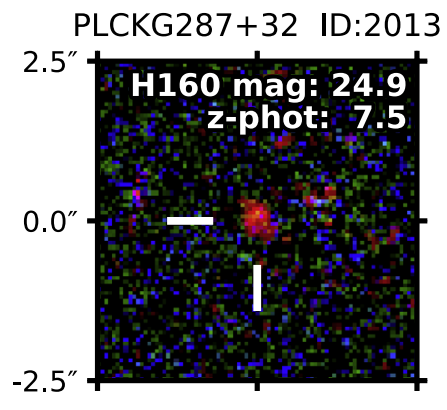

PLCKG287+32 ID:2032

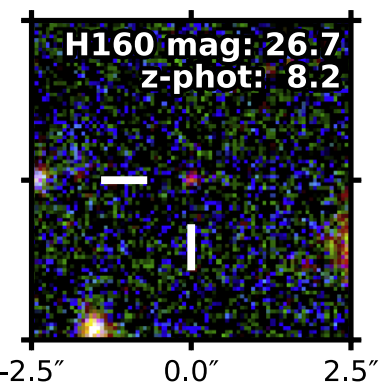

Abell1763 ID:1434

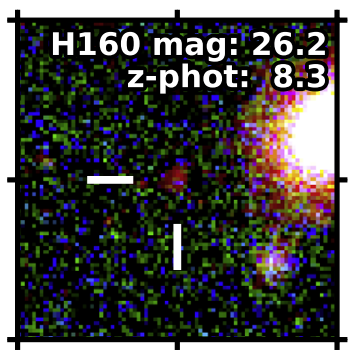

MACS0553-33 ID:219

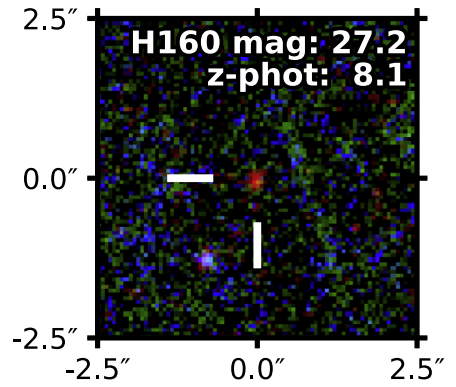

Abells295 ID:568

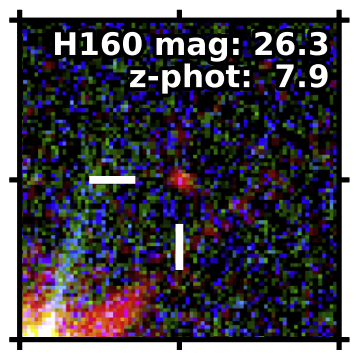

SPT0615-57 ID:1048

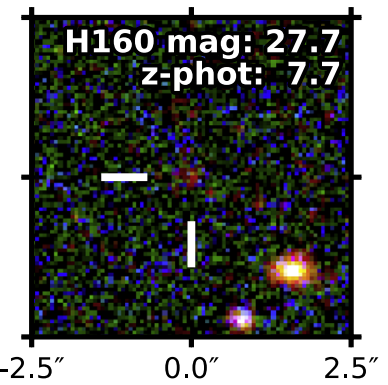

RXC0911+17 ID:143

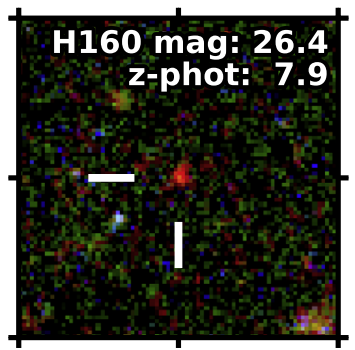

Abell1763 ID:460

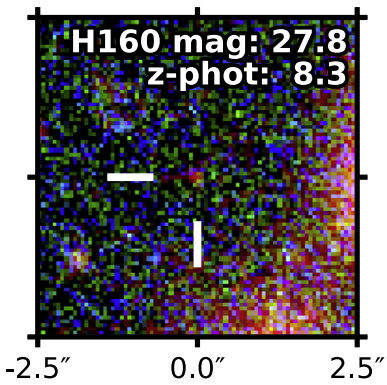

Figure 10. All galaxy candidates from the $z \sim 8$ RELICS sample. Each RGB color image stamp is $5^{\prime \prime} \times 5^{\prime \prime}$ with the red channel as the sum of all IR bands, the G channel as the ACS F814W band, and the B channel the sum of ACS F435W and F606W. The F160W H-band AB magnitude is shown within each stamp, along with the the adopted redshift (see Section 2.3). The cluster name and catalog ID are shown at the top of each stamp.

Table 2

$z \sim 6$ Galaxy Candidates Behind 41 RELICS Clusters

\begin{tabular}{|c|c|c|c|c|c|c|c|}
\hline Object $\mathrm{ID}^{\mathrm{a}}$ & $\alpha_{J 2000}$ & $\delta_{J 2000}$ & $Y_{105}$ & $J_{125}$ & $J H_{140}$ & $H_{160}$ & $z_{\text {phot }}^{b}$ \\
\hline RXS 0603+42-N-1888 & 6:03:10.38 & $+42: 16: 13.38$ & $22.99 \pm 0.02$ & $22.75 \pm 0.02$ & $22.75 \pm 0.02$ & $22.78 \pm 0.01$ & $5.5[5.7]$ \\
\hline RXS 0603+42-S-2085 & $6: 03: 27.44$ & $+42: 10: 56.03$ & $23.61 \pm 0.03$ & $23.19 \pm 0.04$ & $23.21 \pm 0.03$ & $23.21 \pm 0.02$ & $6.0[5.6]$ \\
\hline MACS $0308+26-0904$ & 3:08:53.41 & $+26: 44: 58.94$ & $23.30 \pm 0.03$ & $23.20 \pm 0.05$ & $23.37 \pm 0.04$ & $23.36 \pm 0.03$ & $6.4[6.2]$ \\
\hline RXS 0603+42-S-3486 & 6:03:24.89 & $+42: 10: 16.21$ & $23.78 \pm 0.03$ & $23.40 \pm 0.03$ & $23.47 \pm 0.03$ & $23.41 \pm 0.02$ & $5.5\left[\begin{array}{l}5.7 \\
4.9\end{array}\right]$ \\
\hline RXS 0603+42-N-5737 & 6:03:09.20 & $+42: 14: 52.95$ & $23.69 \pm 0.03$ & $23.55 \pm 0.04$ & $23.48 \pm 0.03$ & $23.48 \pm 0.02$ & $5.7\left[\begin{array}{l}5.8 \\
0.9\end{array}\right]$ \\
\hline RXS 0603+42-S-6059 & 6:03:27.31 & $+42: 09: 00.88$ & $23.79 \pm 0.03$ & $23.53 \pm 0.04$ & $23.53 \pm 0.03$ & $23.54 \pm 0.02$ & $5.7\left[\begin{array}{l}6.0 \\
1.0\end{array}\right]$ \\
\hline RXS 0603+42-N-1050 & 6:03:08.80 & $+42: 16: 35.46$ & $23.88 \pm 0.03$ & $23.65 \pm 0.04$ & $23.58 \pm 0.04$ & $23.57 \pm 0.02$ & $6.1\left[{ }_{5.9}^{6.4}\right]$ \\
\hline SMACS 0723-73-1366 & $7: 23: 15.33$ & $-73: 28: 08.94$ & $24.10 \pm 0.03$ & $23.85 \pm 0.04$ & $23.88 \pm 0.04$ & $23.87 \pm 0.03$ & $5.8[5.5]$ \\
\hline RXC 0600-20-0840 & 6:00:09.55 & $-20: 08: 10.81$ & $24.01 \pm 0.04$ & $23.88 \pm 0.07$ & $23.92 \pm 0.06$ & $23.93 \pm 0.04$ & $6.3\left[\begin{array}{l}6.6 \\
6.1\end{array}\right]$ \\
\hline PLCKG 171-40-0130 & $3: 12: 54.20$ & $+8: 23: 03.88$ & $24.22 \pm 0.10$ & $23.93 \pm 0.12$ & $24.07 \pm 0.11$ & $23.93 \pm 0.06$ & $5.7\left[\begin{array}{l}6.2 \\
0.6\end{array}\right]$ \\
\hline SMACS 0723-73-0711 & $7: 23: 14.52$ & $-73: 27: 10.17$ & $24.21 \pm 0.04$ & $24.08 \pm 0.06$ & $23.99 \pm 0.05$ & $23.96 \pm 0.03$ & $5.5\left[\begin{array}{c}6.0 \\
4.7\end{array}\right]$ \\
\hline PLCK G004-19-0222 & $19: 17: 07.72$ & $-33: 30: 29.49$ & $24.33 \pm 0.06$ & $24.00 \pm 0.07$ & $24.20 \pm 0.07$ & $24.13 \pm 0.05$ & $5.8\left[{ }_{5.4}^{6.1}\right]$ \\
\hline SPT 0615-57-0261 & $6: 15: 49.34$ & $-57: 46: 13.87$ & $24.66 \pm 0.05$ & $24.56 \pm 0.08$ & $24.38 \pm 0.06$ & $24.21 \pm 0.04$ & $5.6\left[\begin{array}{l}6.1 \\
0.1\end{array}\right]$ \\
\hline RXC 2211-03-0196 & $22: 11: 46.82$ & $-3: 49: 00.96$ & $24.43 \pm 0.04$ & $24.18 \pm 0.06$ & $24.36 \pm 0.06$ & $24.23 \pm 0.03$ & $6.2[6.0]$ \\
\hline RXS 0603+42-N-6150 & 6:03:09.77 & $+42: 14: 45.13$ & $24.53 \pm 0.04$ & $24.25 \pm 0.06$ & $24.32 \pm 0.05$ & $24.31 \pm 0.03$ & $5.6[0.9]$ \\
\hline CL 0152-13-1508 & $1: 52: 39.75$ & $-13: 58: 07.12$ & $24.77 \pm 0.07$ & $24.54 \pm 0.04$ & $24.46 \pm 0.08$ & $24.38 \pm 0.03$ & $5.5[5.8]$ \\
\hline MACS 0553-33-1323 & $5: 53: 25.24$ & $-33: 43: 37.01$ & $24.60 \pm 0.05$ & $24.40 \pm 0.06$ & $24.48 \pm 0.06$ & $24.42 \pm 0.04$ & $5.7\left[{ }_{5.3}^{6.1}\right]$ \\
\hline A $2537-0333$ & 23:08:25.00 & $-2: 11: 18.91$ & $24.50 \pm 0.08$ & $24.44 \pm 0.14$ & $24.32 \pm 0.11$ & $24.44 \pm 0.08$ & $5.8[5.4]$ \\
\hline RXC 0142+44-0323 & 1:43:00.70 & $+44: 38: 33.04$ & $24.55 \pm 0.04$ & $24.51 \pm 0.07$ & $24.41 \pm 0.05$ & $24.44 \pm 0.04$ & $5.5[5.1]$ \\
\hline PLCK G004-19-2691 & $19: 17: 03.65$ & $-33: 32: 40.23$ & $24.70 \pm 0.08$ & $24.38 \pm 0.09$ & $24.52 \pm 0.09$ & $24.47 \pm 0.05$ & $6.0[5.6]$ \\
\hline PLCK G004-19-1483 & $19: 17: 08.92$ & $-33: 31: 28.69$ & $24.37 \pm 0.04$ & $24.27 \pm 0.06$ & $24.17 \pm 0.05$ & $24.47 \pm 0.05$ & $5.6[5.3 .9]$ \\
\hline A $2163-1204$ & $16: 15: 40.55$ & $-6: 08: 51.34$ & $24.43 \pm 0.06$ & $24.50 \pm 0.10$ & $24.38 \pm 0.08$ & $24.49 \pm 0.05$ & $5.6[5.2]$ \\
\hline SMACS 0723-73-1430 & $7: 23: 26.29$ & $-73: 28: 19.60$ & $24.73 \pm 0.05$ & $24.58 \pm 0.08$ & $24.64 \pm 0.07$ & $24.51 \pm 0.04$ & $5.5[5.0]$ \\
\hline RXS 0603+42-N-1925 & 6:03:17.84 & $+42: 16: 12.72$ & $24.75 \pm 0.05$ & $24.53 \pm 0.07$ & $24.59 \pm 0.06$ & $24.52 \pm 0.04$ & $5.7\left[{ }_{5.3}^{6.1}\right]$ \\
\hline RXS 0603+42-N-4609 & 6:03:08.79 & $+42: 15: 14.12$ & $24.97 \pm 0.06$ & $24.67 \pm 0.07$ & $24.67 \pm 0.07$ & $24.63 \pm 0.04$ & $6.0\left[\begin{array}{l}6.1 .1 \\
1.1\end{array}\right]$ \\
\hline RXS 0603+42-N-7437 & 6:03:14.48 & $+42: 14: 21.13$ & $24.73 \pm 0.04$ & $24.48 \pm 0.06$ & $24.60 \pm 0.05$ & $24.65 \pm 0.04$ & $5.7[5.5]$ \\
\hline RXS 0603+42-N-4419 & 6:03:06.03 & $+42: 15: 18.05$ & $24.75 \pm 0.06$ & $24.53 \pm 0.09$ & $24.68 \pm 0.08$ & $24.66 \pm 0.05$ & $5.7[5.5]$ \\
\hline A $2537-0397$ & $23: 08: 25.35$ & $-2: 11: 18.13$ & $24.72 \pm 0.04$ & $24.46 \pm 0.06$ & $24.80 \pm 0.07$ & $24.67 \pm 0.04$ & $5.6\left[\begin{array}{c}6.0 \\
5.3\end{array}\right]$ \\
\hline RXC 1514-15-0550 & $15: 15: 02.05$ & $-15: 22: 58.39$ & $24.98 \pm 0.07$ & $24.76 \pm 0.09$ & $24.70 \pm 0.07$ & $24.74 \pm 0.05$ & $5.9[5.4]$ \\
\hline MACS $0308+26-0249$ & 3:08:57.03 & $+26: 46: 07.15$ & $24.71 \pm 0.07$ & $24.75 \pm 0.11$ & $24.78 \pm 0.09$ & $24.78 \pm 0.06$ & $5.6\left[\begin{array}{c}5.8 \\
0.9\end{array}\right]$ \\
\hline MACS $0308+26-0438$ & 3:08:57.19 & $+26: 45: 48.37$ & $24.77 \pm 0.06$ & $24.64 \pm 0.09$ & $24.74 \pm 0.08$ & $24.78 \pm 0.06$ & $6.4[6.9]$ \\
\hline SPT 0615-57-0325 & $6: 15: 54.78$ & $-57: 46: 17.93$ & $25.00 \pm 0.06$ & $24.75 \pm 0.07$ & $24.78 \pm 0.06$ & $24.78 \pm 0.04$ & $6.0\left[{ }_{5.7}^{6.3}\right]$ \\
\hline PLCKG 209+10-0202 & $7: 22: 20.93$ & $+7: 25: 12.38$ & $25.00 \pm 0.06$ & $24.80 \pm 0.09$ & $24.72 \pm 0.07$ & $24.79 \pm 0.05$ & $6.0[5.6]$ \\
\hline
\end{tabular}


Table 2

(Continued)

\begin{tabular}{|c|c|c|c|c|c|c|c|}
\hline Object $\mathrm{ID}^{\mathrm{a}}$ & $\alpha_{J 2000}$ & $\delta_{J 2000}$ & $Y_{105}$ & $J_{125}$ & $J H_{140}$ & $H_{160}$ & $z_{\text {phot }}^{b}$ \\
\hline A $1758-1942$ & $13: 32: 48.03$ & $+50: 31: 06.66$ & $24.69 \pm 0.05$ & $24.82 \pm 0.09$ & $24.77 \pm 0.07$ & $24.82 \pm 0.05$ & $6.1\left[\begin{array}{c}6.2 \\
5.8\end{array}\right]$ \\
\hline RXC 0600-20-1304 & 6:00:07.74 & $-20: 09: 09.41$ & $24.81 \pm 0.06$ & $24.75 \pm 0.09$ & $24.82 \pm 0.09$ & $24.85 \pm 0.06$ & $6.1\left[\begin{array}{c}6.4 \\
6.4\end{array}\right]$ \\
\hline A $2163-1163$ & $16: 15: 46.61$ & $-6: 08: 49.06$ & $24.87 \pm 0.06$ & $24.69 \pm 0.09$ & $24.64 \pm 0.07$ & $24.86 \pm 0.06$ & $6.2[6.0]$ \\
\hline A $2163-1612$ & $16: 15: 40.55$ & $-6: 09: 18.83$ & $24.90 \pm 0.07$ & $24.84 \pm 0.10$ & $24.88 \pm 0.09$ & $24.94 \pm 0.06$ & $6.0[5.6]$ \\
\hline A $295-0250$ & $2: 45: 29.87$ & $-53: 01: 50.40$ & $25.10 \pm 0.07$ & $24.93 \pm 0.11$ & $25.09 \pm 0.11$ & $25.04 \pm 0.07$ & $6.3[6.1]$ \\
\hline RXS $0603+42-S-5318$ & $6: 03: 20.56$ & $+42: 09: 22.18$ & $25.47 \pm 0.09$ & $25.04 \pm 0.10$ & $25.30 \pm 0.11$ & $25.12 \pm 0.06$ & $6.0\left[\begin{array}{l}0.3 .1 \\
6.1\end{array}\right]$ \\
\hline RXC 2211-03-0547 & $22: 11: 43.01$ & $-3: 49: 39.98$ & $25.20 \pm 0.09$ & $25.09 \pm 0.14$ & $25.18 \pm 0.13$ & $25.20 \pm 0.09$ & $5.6\left[\begin{array}{l}5.9 \\
4.8\end{array}\right]$ \\
\hline
\end{tabular}

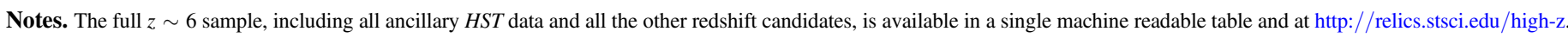
The 40 candidates brightest in F160W are shown here as an example of the format. All magnitudes are given as observed (lensed) isophotal AB magnitudes.

a The online tables use the full cluster name and ID as used by the released RELICS photometric catalogs.

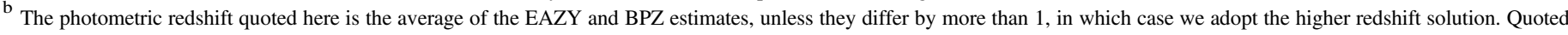
redshift ranges [given in brackets] conservatively span the $95 \%$ confidence intervals from both methods.

(This table is available in its entirety in machine-readable form.)

Table 3

$z \sim 7$ Galaxy Candidates Behind 41 RELICS Clusters

\begin{tabular}{|c|c|c|c|c|c|c|c|}
\hline Object ID & $\alpha_{J 2000}$ & $\delta_{J 2000}$ & $Y_{105}$ & $J_{125}$ & $J H_{140}$ & $H_{160}$ & $z_{\text {phot }}$ \\
\hline RXC0600-20-0178 & 6:00:06.51 & $-20: 07: 12.89$ & $25.01 \pm 0.06$ & $24.67 \pm 0.08$ & $24.55 \pm 0.06$ & $24.42 \pm 0.04$ & $6.6\left[\begin{array}{l}7.1 \\
6.1\end{array}\right]$ \\
\hline MS1008-12-0326 & $10: 10: 31.44$ & $-12: 39: 11.15$ & $25.26 \pm 0.09$ & $24.83 \pm 0.10$ & $24.68 \pm 0.07$ & $24.55 \pm 0.05$ & $7.2\left[\begin{array}{l}7.8 \\
6.6\end{array}\right]$ \\
\hline PLCKG287+32-0698 & 11:50:49.19 & $-28: 04: 14.56$ & $25.11 \pm 0.08$ & $24.95 \pm 0.12$ & $24.98 \pm 0.10$ & $25.00 \pm 0.07$ & $6.8\left[\begin{array}{c}7.5 \\
6.4\end{array}\right]$ \\
\hline PLCKG287+32-0777 & $11: 50: 52.78$ & $-28: 04: 17.49$ & $25.38 \pm 0.09$ & $25.04 \pm 0.11$ & $25.09 \pm 0.10$ & $25.16 \pm 0.07$ & $7.4\left[\begin{array}{c}7.8 \\
6.6\end{array}\right]$ \\
\hline PLCKG287+32-2235 & $11: 50: 51.93$ & $-28: 05: 16.20$ & $25.33 \pm 0.09$ & $25.14 \pm 0.13$ & $25.18 \pm 0.12$ & $25.17 \pm 0.07$ & $7.2\left[\begin{array}{c}7.7 \\
6.5\end{array}\right]$ \\
\hline ABELL3192-1076 & $3: 58: 50.67$ & $-29: 56: 46.38$ & $25.59 \pm 0.09$ & $25.34 \pm 0.14$ & $25.40 \pm 0.12$ & $25.32 \pm 0.08$ & $7.0\left[\begin{array}{l}7.7 \\
6.6\end{array}\right]$ \\
\hline ABELL1300-0226 & 11:31:56.06 & $-19: 54: 54.06$ & $25.97 \pm 0.11$ & $25.58 \pm 0.13$ & $25.92 \pm 0.15$ & $25.62 \pm 0.08$ & $7.0\left[\begin{array}{l}7.7 \\
6.3\end{array}\right]$ \\
\hline RXC0911+17-0419 & 9:11:08.95 & $+17: 46: 53.16$ & $25.36 \pm 0.09$ & $25.65 \pm 0.18$ & $25.25 \pm 0.11$ & $25.64 \pm 0.10$ & $6.8\left[\begin{array}{l}7.2 \\
6.6\end{array}\right]$ \\
\hline ABELL1300-0534 & 11:31:56.49 & $-19: 55: 27.13$ & $26.00 \pm 0.12$ & $25.84 \pm 0.18$ & $25.82 \pm 0.15$ & $25.94 \pm 0.11$ & $6.7\left[\begin{array}{l}7.6 \\
6.2\end{array}\right]$ \\
\hline ABELL3192-0033 & $3: 58: 52.66$ & $-29: 54: 42.78$ & $26.07 \pm 0.11$ & $26.44 \pm 0.28$ & $26.05 \pm 0.17$ & $26.07 \pm 0.12$ & $6.7\left[\begin{array}{c}7.2 \\
6.3\end{array}\right]$ \\
\hline PLCKG287+32-0792 & $11: 50: 48.22$ & $-28: 04: 17.89$ & $26.79 \pm 0.19$ & $26.71 \pm 0.30$ & $26.37 \pm 0.19$ & $26.24 \pm 0.11$ & $7.3\left[\begin{array}{c}7.9 \\
1.2\end{array}\right]$ \\
\hline ACT0102-49-2551 & $1: 02: 54.16$ & $-49: 13: 57.42$ & $25.98 \pm 0.10$ & $25.79 \pm 0.15$ & $25.92 \pm 0.14$ & $26.25 \pm 0.12$ & $6.8\left[\begin{array}{c}7.3 \\
6.5\end{array}\right]$ \\
\hline АCT0102-49-2391 & $1: 02: 53.55$ & $-49: 14: 21.74$ & $26.39 \pm 0.15$ & $25.99 \pm 0.19$ & $26.29 \pm 0.21$ & $26.26 \pm 0.14$ & $6.8\left[\begin{array}{l}7.6 \\
6.2\end{array}\right]$ \\
\hline MS1008-12-0312 & $10: 10: 35.40$ & $-12: 39: 08.82$ & $26.50 \pm 0.15$ & $26.40 \pm 0.22$ & $26.60 \pm 0.22$ & $26.31 \pm 0.12$ & $6.9\left[\begin{array}{l}7.6 \\
1.3\end{array}\right]$ \\
\hline PLCKG287+32-2252 & 11:50:48.94 & $-28: 05: 03.22$ & $26.73 \pm 0.18$ & $26.85 \pm 0.32$ & $26.90 \pm 0.28$ & $26.40 \pm 0.13$ & $6.5\left[\begin{array}{c}7.2 \\
0.8\end{array}\right]$ \\
\hline MS1008-12-0313 & $10: 10: 35.35$ & $-12: 39: 09.07$ & $26.49 \pm 0.16$ & $26.52 \pm 0.26$ & $26.37 \pm 0.19$ & $26.46 \pm 0.14$ & $6.6\left[\begin{array}{l}7.4 \\
1.1\end{array}\right]$ \\
\hline ABELL1763-1500 & $13: 35: 19.44$ & $+40: 59: 19.92$ & $26.55 \pm 0.14$ & $26.84 \pm 0.13$ & $26.35 \pm 0.22$ & $26.50 \pm 0.09$ & $6.5\left[\begin{array}{l}7.2 \\
1.4\end{array}\right]$ \\
\hline SPT0254-58-0476 & $2: 54: 22.50$ & $-58: 56: 58.90$ & $26.52 \pm 0.15$ & $26.84 \pm 0.33$ & $26.49 \pm 0.21$ & $26.50 \pm 0.14$ & $6.5\left[\begin{array}{c}7.3 \\
1.1\end{array}\right]$ \\
\hline $\mathrm{RXC} 0032+18-0571$ & $0: 32: 12.61$ & $+18: 07: 54.78$ & $26.48 \pm 0.16$ & $26.42 \pm 0.26$ & $26.82 \pm 0.29$ & $26.50 \pm 0.16$ & $6.5\left[\begin{array}{c}7.4 \\
5.2\end{array}\right]$ \\
\hline SMACS0723-73-1317 & 7:23:08.60 & $-73: 28: 02.50$ & $26.24 \pm 0.14$ & $26.43 \pm 0.23$ & $26.56 \pm 0.23$ & $26.53 \pm 0.15$ & $6.8\left[\begin{array}{l}7.5 \\
6.3\end{array}\right]$ \\
\hline ABELL520-1342 & 4:54:07.62 & $+2: 54: 13.05$ & $26.86 \pm 0.20$ & $26.53 \pm 0.26$ & $26.36 \pm 0.19$ & $26.61 \pm 0.16$ & $7.2\left[\begin{array}{l}8.1 \\
1.0\end{array}\right]$ \\
\hline CL0152-13-0410 & $1: 52: 43.78$ & $-13: 56: 48.92$ & $26.46 \pm 0.15$ & $26.87 \pm 0.14$ & $26.58 \pm 0.24$ & $26.66 \pm 0.12$ & $6.5\left[\begin{array}{l}6.8 \\
6.0\end{array}\right]$ \\
\hline RXC0142+44-1088 & $1: 42: 51.41$ & $+44: 37: 24.02$ & $27.23 \pm 0.22$ & $27.00 \pm 0.28$ & $27.30 \pm 0.30$ & $26.68 \pm 0.13$ & $7.0\left[\begin{array}{c}7.9 \\
1.1\end{array}\right]$ \\
\hline PLCKG287+32-2457 & $11: 50: 49.68$ & $-28: 05: 24.65$ & $27.15 \pm 0.24$ & $27.46 \pm 0.54$ & $27.63 \pm 0.48$ & $26.69 \pm 0.17$ & $6.7\left[\begin{array}{l}7.7 \\
0.7\end{array}\right]$ \\
\hline CL0152-13-0259 & $1: 52: 43.80$ & $-13: 58: 18.15$ & $26.40 \pm 0.16$ & $26.38 \pm 0.10$ & $26.36 \pm 0.23$ & $26.70 \pm 0.14$ & $6.8\left[\begin{array}{c}7.4 \\
6.4\end{array}\right]$ \\
\hline MS1008-12-0227 & $10: 10: 35.72$ & $-12: 38: 57.83$ & $27.10 \pm 0.24$ & $27.59 \pm 0.55$ & $26.74 \pm 0.24$ & $26.72 \pm 0.17$ & $7.2\left[\begin{array}{l}8.0 \\
0.9\end{array}\right]$ \\
\hline PLCKG004-19-2704 & 19:17:05.31 & $-33: 32: 41.84$ & $26.91 \pm 0.20$ & $26.68 \pm 0.28$ & $27.13 \pm 0.32$ & $26.72 \pm 0.17$ & $6.9\left[\begin{array}{l}7.9 \\
1.1\end{array}\right]$ \\
\hline PLCKG004-19-1983 & 19:17:02.77 & $-33: 31: 49.12$ & $26.55 \pm 0.14$ & $26.71 \pm 0.26$ & $26.72 \pm 0.22$ & $26.75 \pm 0.15$ & $6.5\left[\begin{array}{c}7.3 \\
5.9\end{array}\right]$ \\
\hline ABELL2163-1715 & $16: 15: 43.65$ & $-6: 09: 43.92$ & $26.90 \pm 0.21$ & $26.78 \pm 0.30$ & $26.97 \pm 0.29$ & $26.77 \pm 0.17$ & $6.9\left[\begin{array}{l}7.8 \\
5.6\end{array}\right]$ \\
\hline SMACS0723-73-0599 & $7: 23: 21.67$ & $-73: 26: 59.98$ & $27.17 \pm 0.23$ & $27.30 \pm 0.42$ & $26.77 \pm 0.23$ & $26.82 \pm 0.16$ & $6.5\left[\begin{array}{c}7.8 \\
0.8\end{array}\right]$ \\
\hline PLCKG287+32-0221 & 11:50:51.37 & $-28: 03: 53.21$ & $26.75 \pm 0.28$ & $26.72 \pm 0.50$ & $26.79 \pm 0.40$ & $26.85 \pm 0.31$ & $6.6\left[\begin{array}{l}8.0 \\
1.5\end{array}\right]$ \\
\hline MACS0257-23-0787 & $2: 57: 12.73$ & $-23: 26: 28.09$ & $27.49 \pm 0.27$ & $27.05 \pm 0.32$ & $27.31 \pm 0.33$ & $26.94 \pm 0.17$ & $7.2\left[\begin{array}{l}8.4 \\
1.4\end{array}\right]$ \\
\hline SPT0615-57-0548 & $6: 15: 53.55$ & $-57: 46: 38.70$ & $27.09 \pm 0.20$ & $26.94 \pm 0.29$ & $26.70 \pm 0.21$ & $26.95 \pm 0.17$ & $6.6\left[\begin{array}{c}7.8 \\
5.8\end{array}\right]$ \\
\hline PLCKG287+32-1887 & $11: 50: 45.93$ & $-28: 05: 01.67$ & $27.42 \pm 0.30$ & $27.26 \pm 0.42$ & $27.98 \pm 0.62$ & $27.00 \pm 0.20$ & $7.0\left[\begin{array}{l}8.3 \\
0.9\end{array}\right]$ \\
\hline PLCKG004-19-1156 & 19:17:05.38 & $-33: 31: 15.41$ & $27.21 \pm 0.25$ & $26.93 \pm 0.31$ & $27.05 \pm 0.29$ & $27.01 \pm 0.20$ & $7.1\left[\begin{array}{l}8.1 \\
1.1\end{array}\right]$ \\
\hline MACS0553-33-0463 & $5: 53: 20.41$ & $-33: 42: 12.38$ & $27.36 \pm 0.25$ & $27.34 \pm 0.39$ & $27.32 \pm 0.32$ & $27.15 \pm 0.19$ & $6.5\left[\begin{array}{c}7.8 \\
0.9\end{array}\right]$ \\
\hline MACS0553-33-0865 & $5: 53: 18.49$ & $-33: 42: 46.90$ & $26.96 \pm 0.19$ & $27.10 \pm 0.33$ & $27.54 \pm 0.39$ & $27.16 \pm 0.20$ & $6.7\left[\begin{array}{l}7.5 \\
6.0\end{array}\right]$ \\
\hline ABELL1763-1564 & $13: 35: 19.37$ & $+40: 59: 14.07$ & $27.05 \pm 0.19$ & $27.01 \pm 0.13$ & $27.16 \pm 0.37$ & $27.18 \pm 0.13$ & $6.8\left[\begin{array}{c}7.8 \\
6.0\end{array}\right]$ \\
\hline ABELL1763-0817 & $13: 35: 13.81$ & $+41: 00: 09.68$ & $27.49 \pm 0.25$ & $26.99 \pm 0.11$ & $27.15 \pm 0.35$ & $27.19 \pm 0.12$ & $7.5\left[\begin{array}{l}8.2 \\
5.3\end{array}\right]$ \\
\hline PLCKG287+32-2199 & $11: 50: 52.22$ & $-28: 05: 14.55$ & $27.24 \pm 0.21$ & $27.84 \pm 0.54$ & $27.55 \pm 0.38$ & $27.21 \pm 0.19$ & $6.9\left[\begin{array}{l}7.7 \\
1.0\end{array}\right]$ \\
\hline
\end{tabular}

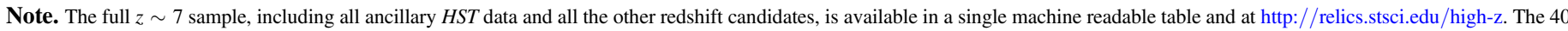
candidates brightest in F160W are shown here as an example of the format. All magnitudes are given as observed (lensed) isophotal AB magnitudes.

(This table is available in its entirety in machine-readable form.) 

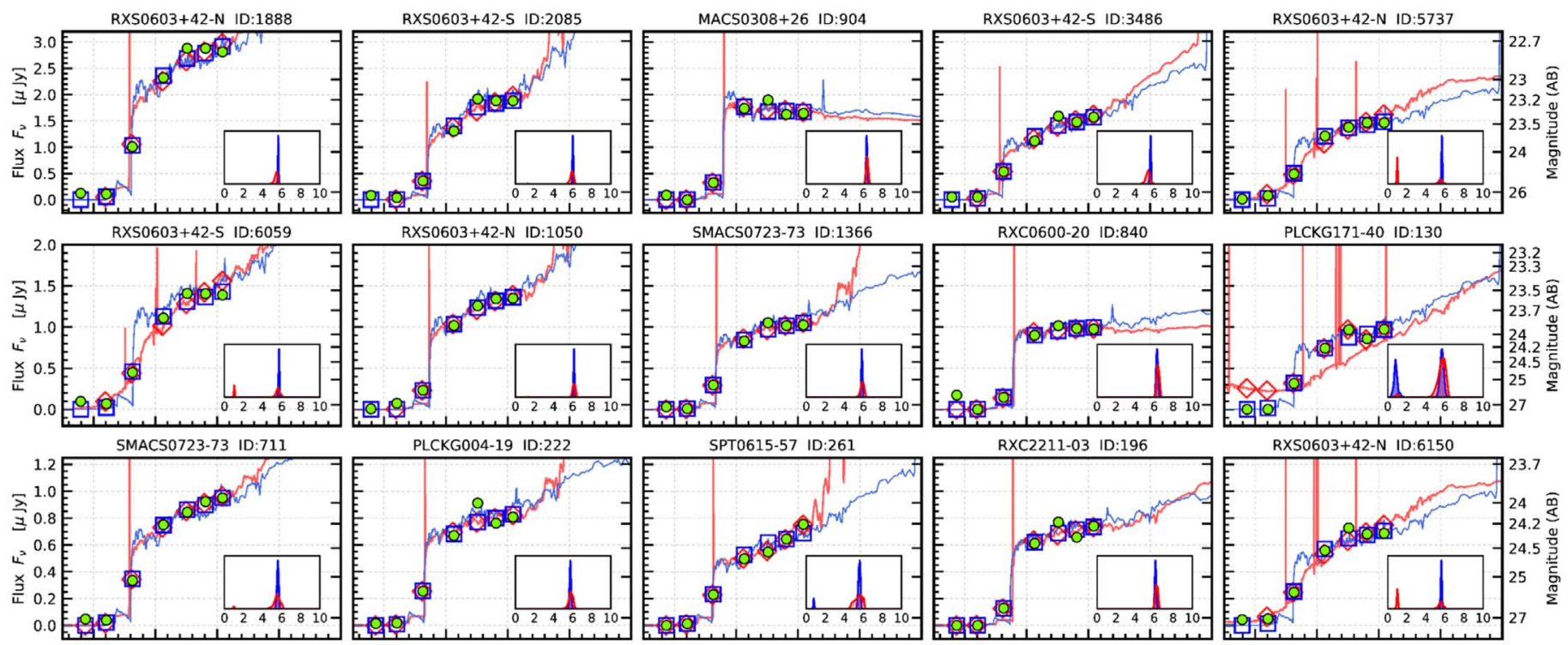

RXC2211-03 ID:196
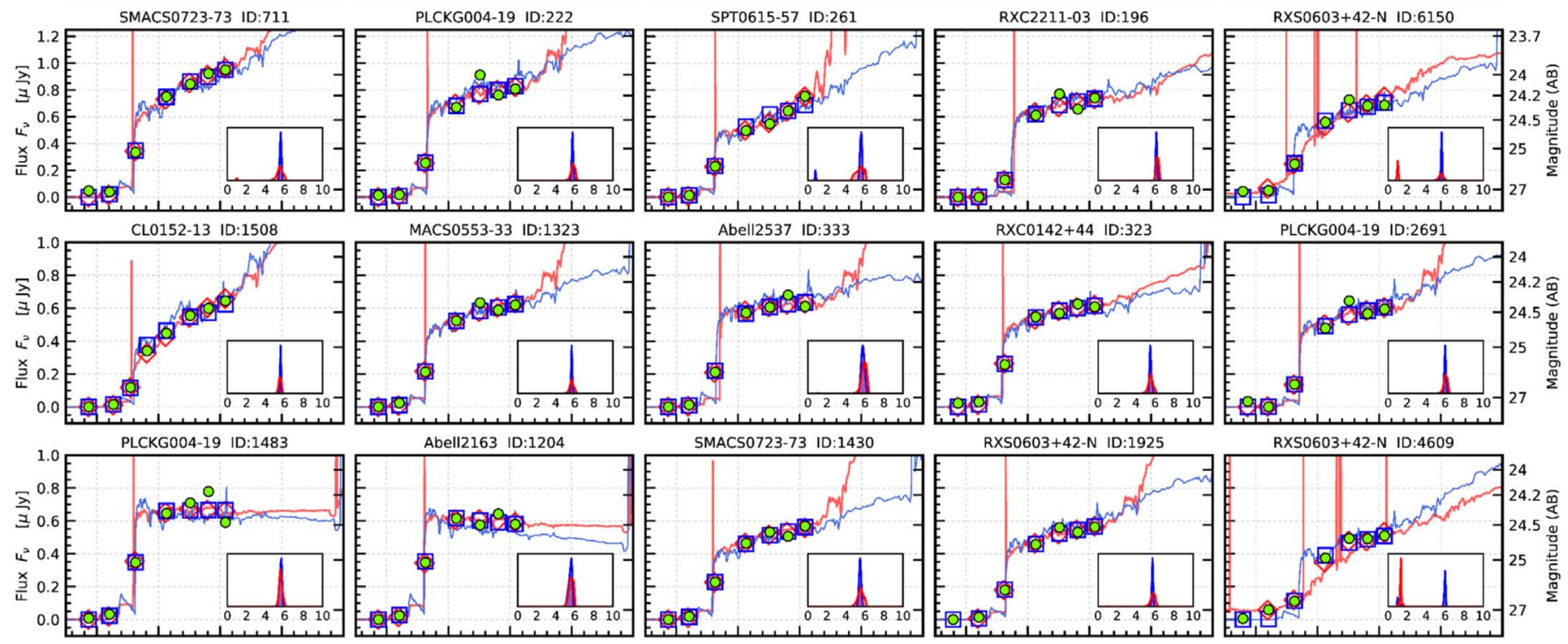

SMACS0723-73 ID:1430
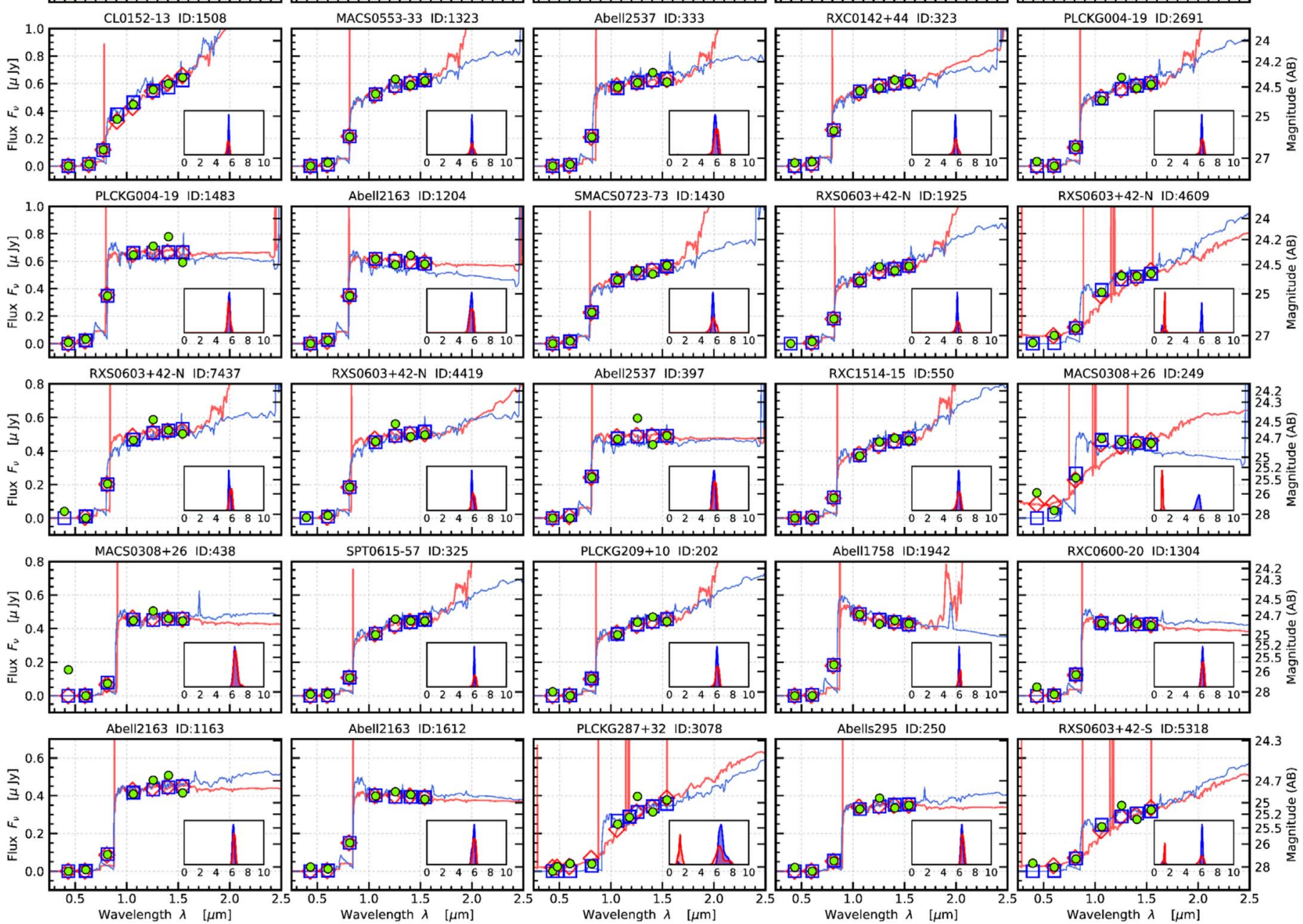

Figure 11. Best-fit SEDs from EAZY (red) and BPZ (blue) for the 50 brightest galaxy candidates from the $z \sim 6$ RELICS sample. The diamonds and squares show the expected fluxes from the model SED for EAZY and BPZ respectively, and the green circles are the RELICS data. The typical photometric uncertainties are about the size of the data points. The top title shows the cluster and catalog ID number of each high- $z$ candidate. The grid of this plot matches to the galaxies shown in Figure 8.

Figures 11,12 , and 13 , respectively. Several objects appear to have red SEDs, but we caution that this is because the restframe optical is unconstrained prior to the inclusion of Spitzer, which makes estimates of physical parameters like stellar mass unreliable. In Tables 2-4 we make available the $z=6,7$, and 8 candidate galaxies from the RELICS survey. We include both the photometric estimates of EAZY and BPZ. The full table is available in machine readable format. 

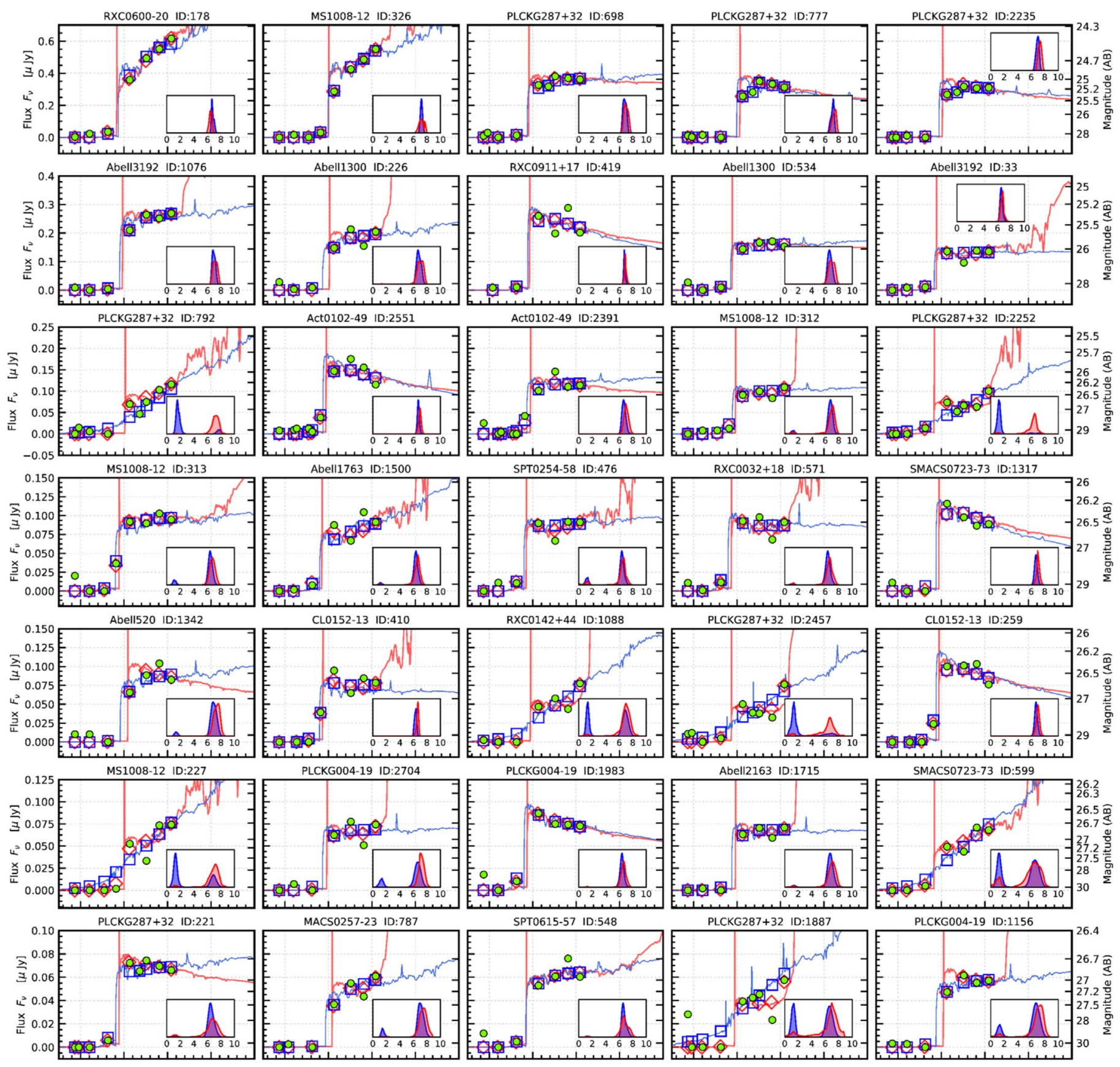

SPT0615-57 ID:548
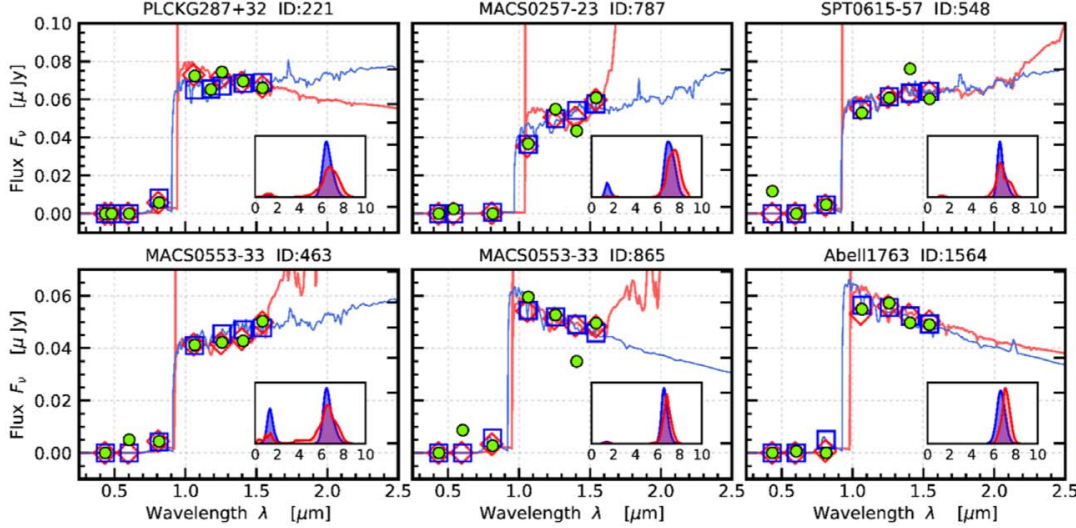

PLCKG287+32 ID:1887
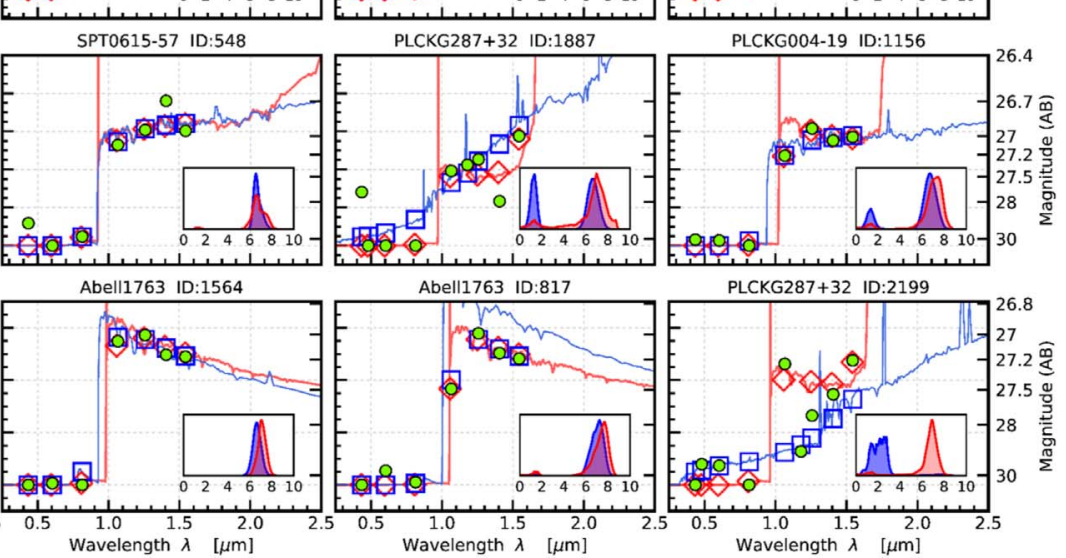

Figure 12. Same as Figure 11, but showing the 40 brightest galaxies from the $z \sim 7$ RELICS sample. The grid of this plot matches to the galaxies shown in Figure 9 .

\section{Conclusions}

We present the candidate high- $z$ galaxies first estimated from RELICS, an HST Treasury Program observing 41 galaxy clusters. We use two independent photometric-redshift fitting codes to determine the redshifts of each galaxy. We also compare the colors of the candidates to those of known dwarf stars, and apply a color selection to remove the most likely contaminants. Furthermore, we conduct a visual inspection of all potential high- $z$ candidates, cleaning the sample of diffraction spikes, misidentified parts of larger galaxies, stars, spurious noise close to the infrared detector edge, transients between epochs, and other image artifacts. 

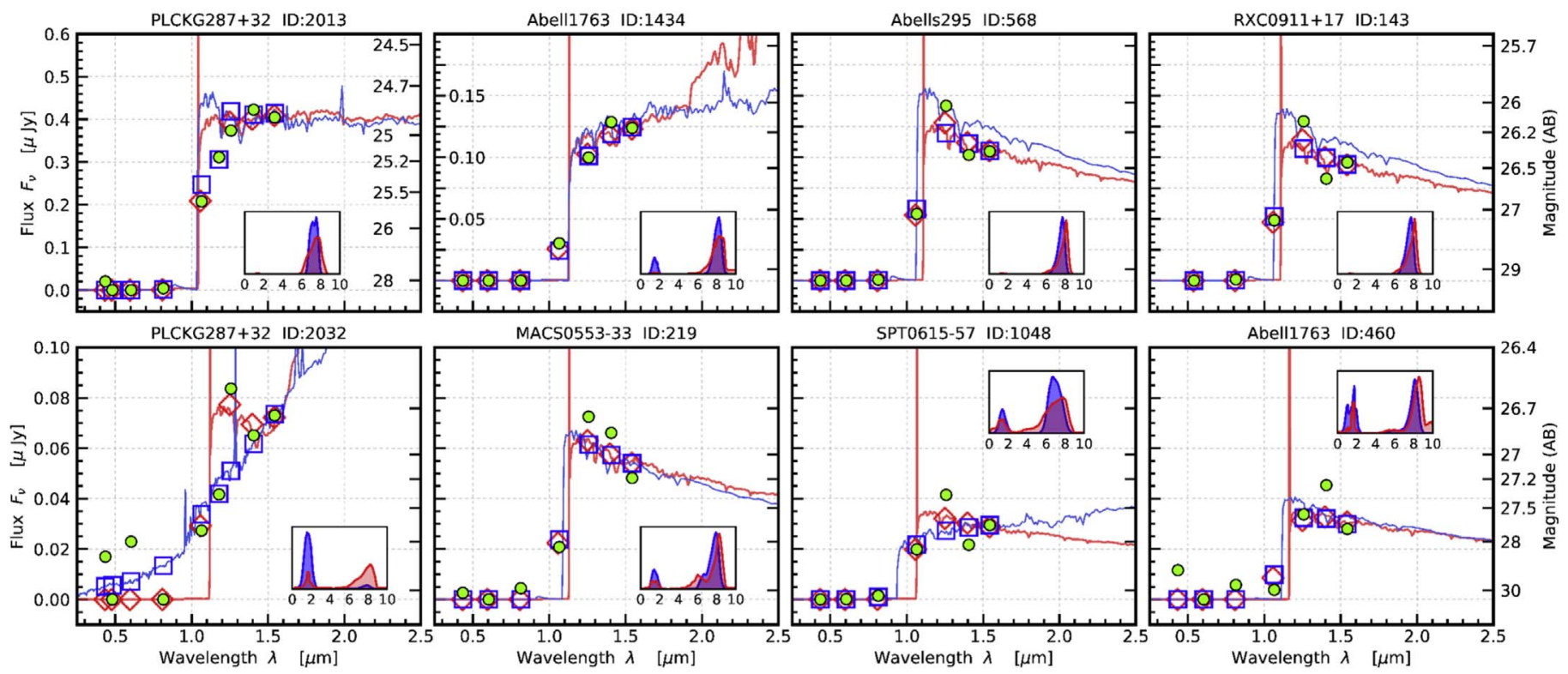

Figure 13. Best-fit SEDs from EAZY (red) and BPZ (blue) for all galaxy candidates from the $z \sim 8$ RELICS sample. The diamonds and squares show the expected fluxes from the model SED for EAZY and BPZ, respectively, and the green circles are the RELICS data. The typical photometric uncertainties are about the size of the data points. The top title shows the cluster and catalog ID number of each high- $z$ candidate. The grid of this plot matches to the galaxies shown in Figure 10 .

Table 4

$z \sim 8$ Galaxy Candidates Behind 41 RELICS Clusters

\begin{tabular}{|c|c|c|c|c|c|c|c|}
\hline Object ID & $\alpha_{J 2000}$ & $\overline{\delta_{J 2000}}$ & $\overline{Y Y_{105}}$ & $\overline{J_{125}}$ & $\overline{J H_{140}}$ & $H_{160}$ & 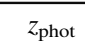 \\
\hline PLCKG 287+32-2013 & $11: 50: 45.07$ & $-28: 04: 33.91$ & $25.61 \pm 0.19$ & $24.97 \pm 0.19$ & $24.84 \pm 0.13$ & $24.88 \pm 0.09$ & $7.5\left[\begin{array}{l}8.3 \\
6.3\end{array}\right.$ \\
\hline A $1763-1434$ & $13: 35: 20.01$ & $+40: 59: 24.65$ & $27.70 \pm 0.43$ & $26.40 \pm 0.10$ & $26.13 \pm 0.22$ & $26.17 \pm 0.08$ & $8.3\left[\begin{array}{l}9.6 \\
1.4\end{array}\right]$ \\
\hline A $295-0568$ & $2: 45: 36.25$ & $-53: 02: 25.87$ & $27.07 \pm 0.22$ & $26.02 \pm 0.17$ & $26.38 \pm 0.19$ & $26.35 \pm 0.12$ & $7.9\left[\begin{array}{l}8.4 \\
6.5\end{array}\right.$ \\
\hline PLCKG 287+32-2032 & $11: 50: 53.42$ & $-28: 05: 06.25$ & $27.81 \pm 0.41$ & $26.59 \pm 0.26$ & $26.87 \pm 0.28$ & $26.74 \pm 0.17$ & $8.2\left[\begin{array}{l}8.7 \\
1.1\end{array}\right]$ \\
\hline MACS 0553-33-0219 & $5: 53: 24.97$ & $-33: 41: 52.61$ & $28.11 \pm 0.41$ & $26.75 \pm 0.23$ & $26.85 \pm 0.21$ & $27.19 \pm 0.18$ & $8.1\left[\begin{array}{l}8.7 \\
1.3\end{array}\right]$ \\
\hline
\end{tabular}

Note. The full $z \sim 8$ sample, including all ancillary HST data and all the other redshift candidates, is available in a single machine readable table and at http://relics. stsci.edu/high-z. All magnitudes are given as observed (lensed) isophotal AB magnitudes.

(This table is available in its entirety in machine-readable form.)

The final sample of candidate high- $z$ galaxies is of comparable size to the CLASH program, despite being significantly more shallow. In particular, we identify several candidates that are among the brightest galaxies at $z \sim 6-8$, as compared with much deeper and wider area surveys. This presents a promising sample for follow-up spectroscopy to study the nebular ionization conditions, Ly $\alpha$ emission, and other galaxy properties into the epoch of reionization.

Finally, we compare the number of galaxies found with the predictions from lensed LFs. We find that our $z \sim 6$ sample agrees with expectations from the literature LFs, while $z \sim 7$ and 8 begin to underproduce the number of candidates compared with expectations from current LFs. The paucity of galaxies from the higher redshift samples could imply the start of an accelerated bright-end evolution in the UV LF, although we await the completion of the Spitzer data and completeness simulations for further confirmation.

We thank Gabriel Brammer for insightful discussions related to this work. This paper is based on observations made with the NASA/ESA Hubble Space Telescope. The Space Telescope
Science Institute (STScI) is operated by the Association of Universities for Research in Astronomy, Inc. (AURA) under NASA contract NAS 5-26555. ACS was developed under NASA contract NAS 5-32864. The Spitzer Space Telescope is operated by the Jet Propulsion Laboratory, California Institute of Technology under a contract with NASA. These observations are associated with program GO-14096. Archival data are associated with programs GO-9270, GO-12166, GO-12477, and GO-12253. Some of the data presented in this paper were obtained from the Mikulski Archive for Space Telescopes (MAST). This research made use of Photutils, an Astropy package for detection and photometry of astronomical sources (Bradley et al. 2019). This research made use of Astropy, a community-developed core Python package for Astronomy (The Astropy Collaboration et al. 2018). This work was performed under the auspices of the U.S. Department of Energy by Lawrence Livermore National Laboratory under Contract DE-AC52-07NA27344. F.A.-S. acknowledges support from Chandra grant G03-14131X. M.B. and V.S. acknowledge support by NASA through ADAP grant 80NSSC18K0945, NASA/HST through HST-GO-14096, HST-GO-13666 and two 
awards issued by Spitzer/JPL/Caltech associated with SRELICS_DEEP and SRELICS programs.

\section{ORCID iDs}

Brett Salmon (1) https://orcid.org/0000-0002-7453-7279 Dan Coe (i) https://orcid.org/0000-0001-7410-7669 Larry Bradley (i) https://orcid.org/0000-0002-7908-9284 Rychard Bouwens (i) https://orcid.org/0000-0002-4989-2471 Marusa Bradač (i) https://orcid.org/0000-0001-5984-0395 Kuang-Han Huang (i) https://orcid.org/0000-0001-7826-6448 Pascal A. Oesch (10 https://orcid.org/0000-0001-5851-6649 Keren Sharon (1) https://orcid.org/0000-0002-7559-0864 Michele Trenti (1) https://orcid.org/0000-0001-9391-305X Felipe Andrade-Santos (1) https://orcid.org/0000-0002-8144-9285 Daniela Carrasco (i) https://orcid.org/0000-0002-3772-0330 William Dawson (16) https://orcid.org/0000-0003-0248-6123 Austin Hoag (i) https://orcid.org/0000-0001-8989-2567 Traci Lin Johnson (ii) https://orcid.org/0000-0002-8829-5303 Daniel Lam (1) https://orcid.org/0000-0002-6536-5575 Ramesh Mainali (1) https://orcid.org/0000-0003-0094-6827 Rachel Paterno-Mahler (i) https://orcid.org/0000-0003-3653-3741 Steven A. Rodney (1) https://orcid.org/0000-0003-1947-687X Victoria Strait (1) https://orcid.org/0000-0002-6338-7295 Keiichi Umetsu (1) https://orcid.org/0000-0002-7196-4822 Benedetta Vulcani (i) https://orcid.org/0000-0003-0980-1499 Adi Zitrin (i) https://orcid.org/0000-0002-0350-4488

\section{References}

Alavi, A., Siana, B., Richard, J., et al. 2016, ApJ, 832, 56 Applegate, D. E., von der Linden, A., Kelly, P. L., et al. 2014, MNRAS, 439, 48 Atek, H., Richard, J., Jauzac, M., et al. 2015a, ApJ, 814, 69 Atek, H., Richard, J., Kneib, J.-P., et al. 2014, ApJ, 786, 60 Atek, H., Richard, J., Kneib, J.-P., et al. 2015b, ApJ, 800, 18 Beckwith, S. V. W., Stiavelli, M., Koekemoer, A. M., et al. 2006, AJ, 132, 1729

Benítez, N. 2000, ApJ, 536, 571

Benítez, N., Ford, H., Bouwens, R., et al. 2004, ApJS, 150, 1

Bertin, E., \& Arnouts, S. 1996, A\&AS, 117, 393

Bleem, L. E., Stalder, B., de Haan, T., et al. 2015, ApJS, 216, 27

Bouwens, R. J., Bradley, L., Zitrin, A., et al. 2014, ApJ, 795, 126

Bouwens, R. J., Illingworth, G. D., Oesch, P. A., et al. 2011, ApJ, 737, 90 Bouwens, R. J., Illingworth, G. D., Oesch, P. A., et al. 2015, ApJ, 803, 34 Bouwens, R. J., Illingworth, G. D., Oesch, P. A., et al. 2017a, ApJ, 843, 41 Bouwens, R. J., Oesch, P. A., Illingworth, G. D., Ellis, R. S., \& Stefanon, M. 2017b, ApJ, 843, 129

Bowler, R. A. A., Dunlop, J. S., McLure, R. J., et al. 2012, MNRAS, 426, 2772 Bowler, R. A. A., Dunlop, J. S., McLure, R. J., et al. 2014, MNRAS, 440, 2810 Bowler, R. A. A., Dunlop, J. S., McLure, R. J., et al. 2015, MNRAS, 452, 1817 Bowler, R. A. A., Dunlop, J. S., McLure, R. J., \& McLeod, D. J. 2017 MNRAS, 466, 3612

Bradley, L., Sipocz, B., Robitaille, T., et al. 2019, astropy/photutils: v0.6, Zenodo, doi:10.5281/zenodo.2533376

Bradley, L. D., Bouwens, R. J., Ford, H. C., et al. 2008, ApJ, 678, 647

Bradley, L. D., Trenti, M., Oesch, P. A., et al. 2012, ApJ, 760, 108

Bradley, L. D., Zitrin, A., Coe, D., et al. 2014, ApJ, 792, 76

Brammer, G. B., van Dokkum, P. G., \& Coppi, P. 2008, ApJ, 686, 1503

Brinchmann, J., Inami, H., Bacon, R., et al. 2017, A\&A, 608, A3

Bullock, J. S., Kolatt, T. S., Sigad, Y., et al. 2001, MNRAS, 321, 559

Bunker, A. J., Stanway, E. R., Ellis, R. S., \& McMahon, R. G. 2004, MNRAS, 355,374

Calvi, V., Trenti, M., Stiavelli, M., et al. 2016, ApJ, 817, 120

Cerny, C., Sheren, K., Coe, D., et al. 2017, ApJ, submitted

Coe, D., Benítez, N., Sánchez, S. F., et al. 2006, AJ, 132, 926

Coe, D., Bradley, L., \& Zitrin, A. 2015, ApJ, 800, 84

Coe, D., Salmon, B., Bradac, M., et al. 2019, ApJ, 884, 85

Coe, D., Zitrin, A., Carrasco, M., et al. 2013, ApJ, 762, 32

Cushing, M. C., Rayner, J. T., \& Vacca, W. D. 2005, ApJ, 623, 1115

Ebeling, H., Edge, A. C., \& Henry, J. P. 2001, ApJ, 553, 668
Ellis, R. S., McLure, R. J., Dunlop, J. S., et al. 2013, ApJL, 763, L7 Erb, D. K., Pettini, M., Shapley, A. E., et al. 2010, ApJ, 719, 1168 Finkelstein, S. L. 2016, PASA, 33, e037

Finkelstein, S. L., Papovich, C., Ryan, R. E., et al. 2012, ApJ, 758, 93 Finkelstein, S. L., Ryan, R. E., Jr., Papovich, C., et al. 2015, ApJ, 810, 71 Fioc, M., \& Rocca-Volmerange, B. 1997, A\&A, 326, 950

Franx, M., Illingworth, G. D., Kelson, D. D., van Dokkum, P. G., \& Tran, K.-V. 1997, ApJL, 486, L75

Gonzaga, S., et al. 2012, The DrizzlePac Handbook (Baltimore, MD: STScI) Grogin, N. A., Kocevski, D. D., Faber, S. M., et al. 2011, ApJS, 197, 35 Harikane, Y., Ouchi, M., Ono, Y., et al. 2018, PASJ, 70, S11

Hashimoto, T., Laporte, N., Mawatari, K., et al. 2018, Natur, 557, 392 Hasselfield, M., Hilton, M., Marriage, T. A., et al. 2013, JCAP, 7, 008 Hoag, A., Bradač, M., Trenti, M., et al. 2017, NatAs, 1, 0091 Hoekstra, H., Herbonnet, R., Muzzin, A., et al. 2015, MNRAS, 449, 685 Illingworth, G. D., Magee, D., Oesch, P. A., et al. 2013, ApJS, 209, 6 Ishigaki, M., Kawamata, R., Ouchi, M., et al. 2015, ApJ, 799, 12 Ishigaki, M., Kawamata, R., Ouchi, M., et al. 2018, ApJ, 854, 73 Jones, T. A., Ellis, R. S., Schenker, M. A., \& Stark, D. P. 2013, ApJ, 779, 52 Kawamata, R., Ishigaki, M., Shimasaku, K., Oguri, M., \& Ouchi, M. 2015, ApJ, 804, 103

Koekemoer, A. M., Ellis, R. S., McLure, R. J., et al. 2013, ApJS, 209, 3 Koekemoer, A. M., Faber, S. M., Ferguson, H. C., et al. 2011, ApJS, 197, 36 Konno, A., Ouchi, M., Shibuya, T., et al. 2018, PASJ, 70, S16 Kuhlen, M., \& Faucher-Giguère, C.-A. 2012, MNRAS, 423, 862 Lam, D., Bouwens, R. J., Labbé, I., et al. 2019, A\&A, 627, A164 Livermore, R. C., Finkelstein, S. L., \& Lotz, J. M. 2017, ApJ, 835, 113 Livermore, R. C., Trenti, M., Bradley, L. D., et al. 2018, ApJL, 861, L17 Lotz, J. M., Koekemoer, A., Coe, D., et al. 2017, ApJ, 837, 97 Madau, P. 1995, ApJ, 441, 18

Madau, P., Haardt, F., \& Rees, M. J. 1999, ApJ, 514, 648

Mainali, R., Kollmeier, J. A., Stark, D. P., et al. 2017, ApJL, 836, L14

Mantz, A., Allen, S. W., Ebeling, H., Rapetti, D., \& Drlica-Wagner, A. 2010 MNRAS, 406, 1773

Maraston, C. 2005, MNRAS, 362, 799

Matthee, J., Sobral, D., Darvish, B., et al. 2017, MNRAS, 472, 772

McCracken, H. J., Milvang-Jensen, B., Dunlop, J., et al. 2012, A\&A, 544, A156

McLeod, D. J., McLure, R. J., \& Dunlop, J. S. 2016, MNRAS, 459, 3812 McLure, R. J., Dunlop, J. S., Bowler, R. A. A., et al. 2013, MNRAS, 432, 2696 Meneghetti, M., Natarajan, P., Coe, D., et al. 2017, MNRAS, 472, 3177 Merlin, E., Bourne, N., Castellano, M., et al. 2016, A\&A, 595, A97 Neto, A. F., Gao, L., Bett, P., et al. 2007, MNRAS, 381, 1450 Oesch, P. A., Bouwens, R. J., Illingworth, G. D., et al. 2012, ApJ, 759, 135 Oesch, P. A., Bouwens, R. J., Illingworth, G. D., et al. 2015, ApJ, 808, 104 Oesch, P. A., Brammer, G., van Dokkum, P. G., et al. 2016, ApJ, 819, 129 Oesch, P. A., Carollo, C. M., Stiavelli, M., et al. 2009, ApJ, 690, 1350

Oke, J. B., \& Gunn, J. E. 1983, ApJ, 266, 713

Ono, Y., Ouchi, M., Harikane, Y., et al. 2018, PASJ, 70, S10 Ouchi, M., Harikane, Y., Shibuya, T., et al. 2018, PASJ, 70, S13

Pentericci, L., Vanzella, E., Fontana, A., et al. 2014, ApJ, 793, 113

Piffaretti, R., Arnaud, M., Pratt, G. W., Pointecouteau, E., \& Melin, J.-B. 2011 A\&A, 534, A109

Planck Collaboration, Ade, P. A. R., Aghanim, N., et al. 2016, A\&A, 594, A27

Postman, M., Coe, D., Benítez, N., et al. 2012, ApJS, 199, 25

Rayner, J. T., Cushing, M. C., \& Vacca, W. D. 2009, ApJS, 185, 289

Rigby, J. R., Bayliss, M. B., Gladders, M. D., et al. 2015, ApJL, 814, L6

Robertson, B. E., Ellis, R. S., Furlanetto, S. R., \& Dunlop, J. S. 2015, ApJL, 802, L19

Schechter, P. 1976, ApJ, 203, 297

Schenker, M. A., Stark, D. P., Ellis, R. S., et al. 2012, ApJ, 744, 179

Schlafly, E. F., \& Finkbeiner, D. P. 2011, ApJ, 737, 103

Schmidt, K. B., Treu, T., Trenti, M., et al. 2014, ApJ, 786, 57

Scoville, N., Aussel, H., Brusa, M., et al. 2007, ApJS, 172, 1

Sereno, M. 2015, MNRAS, 450, 3665

Shibuya, T., Ouchi, M., Harikane, Y., et al. 2018a, PASJ, 70, S15

Shibuya, T., Ouchi, M., Konno, A., et al. 2018b, PASJ, 70, S14

Stark, D. P. 2016, ARA\&A, 54, 761

Stark, D. P., Ellis, R. S., Charlot, S., et al. 2017, MNRAS, 464, 469

Stark, D. P., Ellis, R. S., Chiu, K., Ouchi, M., \& Bunker, A. 2010, MNRAS, 408, 1628

Stark, D. P., Richard, J., Charlot, S., et al. 2015a, MNRAS, 450, 1846 Stark, D. P., Richard, J., Siana, B., et al. 2014, MNRAS, 445, 3200 Stark, D. P., Walth, G., Charlot, S., et al. 2015b, MNRAS, 454, 1393 Stefanon, M., Labbé, I., Bouwens, R. J., et al. 2017, ApJ, 851, 43 Tilvi, V., Papovich, C., Tran, K.-V. H., et al. 2013, ApJ, 768, 56 
The Astropy Collaboration, Price-Whelan, A. M., Sipőcz, B. M., et al. 2018, AJ, 156, 123

Trenti, M., Bradley, L. D., Stiavelli, M., et al. 2011, ApJL, 727, L39

Umetsu, K., Medezinski, E., Nonino, M., et al. 2014, ApJ, 795, 163

Vanzella, E., Calura, F., Meneghetti, M., et al. 2017, MNRAS, 467, 4304

Vanzella, E., De Barros, S., Cupani, G., et al. 2016, ApJL, 821, L27

von der Linden, A., Allen, M. T., Applegate, D. E., et al. 2014, MNRAS, 439, 2
Wen, Z. L., Han, J. L., \& Liu, F. S. 2012, ApJS, 199, 34

Wong, K. C., Zabludoff, A. I., Ammons, S. M., et al. 2013, ApJ, 769, 52

Wright, E. L., Eisenhardt, P. R. M., Mainzer, A. K., et al. 2010, AJ, 140, 1868

Yan, H., Windhorst, R. A., \& Cohen, S. H. 2003, ApJL, 585, L93

Zheng, W., Postman, M., Zitrin, A., et al. 2012, Natur, 489, 406

Zitrin, A., Moustakas, J., Bradley, L., et al. 2012, ApJL, 747, L9

Zitrin, A., Zheng, W., Broadhurst, T., et al. 2014, ApJL, 793, L12 